\title{
Energetics of hydrogen coverage on group VIII transition metal surfaces and a kinetic model for adsorption/desorption
}

\author{
Francesco Faglionia) \\ Dipartimento di Chimica, Università degli Studi, Via G. Campi 183, 41100 Modena, Italy \\ William A. Goddard III ${ }^{\text {b) }}$ \\ Materials and Process Simulation Center (139-74), California Institute of Technology, \\ Pasadena, California 91125
}

(Received 7 August 2003; accepted 21 September 2004; published online 13 December 2004)

\begin{abstract}
We determined the binding energy of hydrogen to the closest packed surface for all nine group VIII transition metals as a function of surface coverage using quantum mechanics (density functional theory with the generalized gradient approximation) with periodic boundary conditions. The study provides a systematic comparison of the most stable surfaces of the nine group VIII transition metals, leading to results consistent with available surface science studies. We then use these to develop a simple thermodynamic model useful in estimating the surface coverage under typical heterogeneous catalysis conditions and compare these results to temperature programmed desorption experiments. (c) 2005 American Institute of Physics. [DOI: 10.1063/1.1814938]
\end{abstract}

\section{INTRODUCTION}

Many of the group VIII transition metals play an important role in industrially important heterogeneous catalysts for a broad range of hydrocarbon transformations. ${ }^{1,2}$ Consequently the behavior of their surfaces has been object of intense study for several decades using techniques ranging from surface science studies in ultrahigh vacuum to kinetic studies to theoretical simulations. However, due to the complexity of heterogeneous reactions under catalytic conditions, there remains considerable uncertainty regarding the chemical behavior of the surface. Indeed even the nature of the simplest adsorbates is not well characterized. In particular, a central role in hydrocarbon reactions and rearrangements on surfaces is played by the behavior of hydrogen under reaction conditions of temperature and pressure, but it is difficult to obtain either experimental or theoretical predictions for these reaction conditions. Indeed even a systematic comparison for different metals is hampered by the wide range of techniques and coverage conditions used by the various groups. Reviews of hydrogen chemistry on transition metal surfaces are available in the literature. ${ }^{3-5}$

In this paper we report consistent computational studies of hydrogen adsorption on the most stable surfaces of all nine group VIII transition metals. The aim of the study is to provide a systematic comparison of the hydrogen binding energy on the surfaces considered at varying levels of coverage in order to provide a consistent set of results to be used in interpreting both catalysis experiments and surface science studies. Although subsurface hydrogen may participate in some surface reactions, we consider here only surface adsorption.

In Sec. II we describe the computational details and the main approximations used with an estimate of the error bars

\footnotetext{
a)Electronic mail: cecco@unimo.it

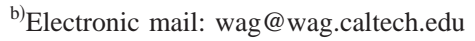

associated with our result. In Sec. III we report the computed binding energies and geometries and compare with available results from other groups. In Sec. IV we discuss our results in terms of three simple statistical thermodynamics treatments of the adsorbed hydrogen. Specific examples are provided in the case of Ir to illustrate a comparison with surface science results. Here, we also review the experimental literature and compare to our predictions where possible.

\section{COMPUTATIONAL DETAILS}

\section{A. Quantum mechanics}

We used the generalized gradient approximation (GGA) density functional developed by Perdew and Wang ${ }^{6}$ with a periodic plane-wave basis set to describe the wave function. All computations were performed using CASTEP (Ref. 7) via the CERIUS2 interface. ${ }^{8}$ Recpot $^{9}$ effective core potential (ECP) was used on all metal atoms and Troullier-Martins ECP (Refs. 10 and 11) on hydrogen, as implemented in CASTEP. The cutoff energy for plane wave expansion was set at $600 \mathrm{eV}$ for all systems. This value was selected according to recommendations for the ECPs and is expected to provide consistently good quality results. We kept the cutoff constant for all nine systems to simplify comparison of numerical results.

\section{B. Surface calculations}

All metal atoms were held fixed at the experimental bulk geometry with only the position of the adsorbed hydrogen optimized. Based on our testing and on published results ${ }^{12,13}$ we expect relaxation to affect binding energies by up to 2 $\mathrm{kcal} / \mathrm{mol}$. We did not consider subsurface adsorption.

We carried out calculations on platinum and iridium surfaces for various number of layers in the slab and found reasonable convergence in the hydrogen binding energy and equilibrium geometry starting at four layers. Specifically, 
adding a fifth layer changed the hydrogen binding energy by less than $0.3 \mathrm{kcal} / \mathrm{mol}$ for Ir and $0.1 \mathrm{kcal} / \mathrm{mol}$ for Pt. Hence, we adopted a four-layer slab for all metallic surfaces. For the vacuum between slabs we used an interlayer distance equivalent to six layers at the bulk geometry (five layers of vacuum). Hydrogen was allowed to adsorb only on one side of the slab.

For the surface unit cells (SUC) we used both $1 \times 1$ and $\sqrt{3} \times \sqrt{3} R\left(30^{\circ}\right)$. The convergence of the total energy and the binding energy with the number of $k$ points was tested in the case of $\mathrm{Pt}(111)$ with $1 \times 1$ SUC. We found that a $12 \times 12$ Monkhorst-Pack grid in the plane of the surface yields energies within $0.016 \mathrm{eV}(0.38 \mathrm{kcal} / \mathrm{mol})$ of the value obtained with the $20 \times 20$ grid. Hence we used the $12 \times 12$ MonkhorstPack grids on $1 \times 1$ SUC. Accordingly, for the $\sqrt{3}$ $\times \sqrt{3} R\left(30^{\circ}\right)$ SUC we used the $7 \times 7$ grids. One $k$ point was used for the direction normal to the surface.

Spin polarization of the wave function was not allowed. We tested the magnitude of this approximation by computing the fcc $\mathrm{H}$ binding energy on $\mathrm{Ni}(111)$ at monolayer (ML) coverage both with and without spin polarization. Due to software restrictions, to perform this task we used ultrasoft potentials ${ }^{14}$ with core correction on the metals and an energy cutoff of $340 \mathrm{eV}$. The computed values are 67.8 and 66.2 $\mathrm{kcal} / \mathrm{mol}$ with and without spin polarization, respectively. The effect of spin polarization is thus of $1.6 \mathrm{kcal} / \mathrm{mol}$, which is smaller than the error bar associated with the choice of the ECP (vide infra).

\section{Kinetics simulations}

Adsorption and desorption kinetics simulations were performed by numerical integration of the Eqs. (36), (38), and (39). All simulated kinetics proved independent from the numerical integration technique used provided that sufficiently small time steps were used. The results reported were obtained with standard fourth-order Runge-Kutta equations using variable time steps. The initial time step was set at $0.01 \mathrm{~s}$. The software used to do these simulations is available in the supplementary material.

\section{RESULTS}

In order to understand hydrogen binding to closest packed surfaces, it is essential to establish the binding geometry and to estimate the barrier for surface diffusion. To study this we used the $1 \times 1$ SUC to compute hydrogen binding energy (BE) at different surface sites for ML coverages. We then selected the most stable sites to investigate how the BE depends on coverage.

The computed hydrogen $\mathrm{BE}$ and equilibrium distance from the surface for the high symmetry positions on the closest packed surfaces considered are reported in Table I. In the case of $\mathrm{Fe}$ and $\mathrm{Co}$, we considered both the phases found at room temperature and pressure (bcc and hcp, respectively) and the two closest packed phases stable under more drastic conditions (fcc). To compute the BE, we assumed the energy of gas phase hydrogen to be the exact value for the Hamiltonian used, i.e., -0.5 hartree $(-13.60570 \mathrm{eV})$. The BE reported in Table I do not include zero point energy (ZPE)
TABLE I. Computed hydrogen binding energy to metal surfaces at different surface sites. The data refer to $1 \mathrm{ML}$ coverage. See text for computational details.

\begin{tabular}{|c|c|c|c|}
\hline Surface & Site & $\begin{array}{c}D_{e} \\
(\mathrm{kcal} / \mathrm{mol})\end{array}$ & $\begin{array}{c}\text { Height } \\
(\AA)\end{array}$ \\
\hline \multirow{4}{*}{$\begin{array}{l}\text { Fe-bcc } \\
(110)\end{array}$} & cap & 65.3 & 0.981 \\
\hline & bridge 1 & 60.7 & 0.968 \\
\hline & bridge2 & 61.3 & 1.192 \\
\hline & top & 48.1 & 1.577 \\
\hline \multirow{4}{*}{$\begin{array}{l}\text { Fe-fcc } \\
(111)\end{array}$} & $\mathrm{fcc}$ & 67.1 & 0.972 \\
\hline & hcp & 66.5 & 0.961 \\
\hline & top & 46.4 & 1.573 \\
\hline & bridge & 61.3 & 1.124 \\
\hline \multirow{4}{*}{$\begin{array}{l}\text { Ru-hep } \\
(001)\end{array}$} & $\mathrm{fcc}$ & 61.8 & 1.090 \\
\hline & hcp & 61.7 & 1.079 \\
\hline & top & 49.7 & 1.652 \\
\hline & bridge & 58.0 & 1.233 \\
\hline \multirow{4}{*}{$\begin{array}{l}\text { Os-hcp } \\
(001)\end{array}$} & fcc & 62.2 & 1.081 \\
\hline & hcp & 61.3 & 1.077 \\
\hline & top & 60.1 & 1.638 \\
\hline & bridge & 60.9 & 1.223 \\
\hline \multirow{4}{*}{$\begin{array}{l}\text { Co-hcp } \\
(001)\end{array}$} & fcc & 61.3 & 1.009 \\
\hline & hcp & 60.3 & 0.990 \\
\hline & top & 45.2 & 1.551 \\
\hline & bridge & 56.6 & 1.139 \\
\hline \multirow{4}{*}{$\begin{array}{l}\text { Co-fcc } \\
(111)\end{array}$} & fcc & 61.6 & 0.999 \\
\hline & hcp & 60.9 & 0.997 \\
\hline & top & 45.2 & 1.560 \\
\hline & bridge & 56.9 & 1.138 \\
\hline \multirow{4}{*}{$\begin{array}{l}\text { Rh-fcc } \\
(111)\end{array}$} & fcc & 58.7 & 1.042 \\
\hline & hcp & 57.7 & 1.021 \\
\hline & top & 49.6 & 1.616 \\
\hline & bridge & 55.8 & 1.184 \\
\hline \multirow{4}{*}{$\begin{array}{l}\text { Ir-fcc } \\
(111)\end{array}$} & fcc & 60.4 & 1.040 \\
\hline & hcp & 59.2 & 1.035 \\
\hline & top & 61.8 & 1.587 \\
\hline & bridge & 59.6 & 1.171 \\
\hline \multirow{4}{*}{$\begin{array}{l}\text { Ni-fcc } \\
(111)\end{array}$} & fcc & 60.9 & 0.955 \\
\hline & hcp & 60.1 & 0.962 \\
\hline & top & 44.1 & 1.525 \\
\hline & bridge & 55.9 & 1.090 \\
\hline \multirow{4}{*}{$\begin{array}{l}\text { Pd-fcc } \\
(111)\end{array}$} & fcc & 59.3 & 0.918 \\
\hline & hcp & 57.4 & 0.891 \\
\hline & top & 45.7 & 1.594 \\
\hline & bridge & 54.1 & 1.088 \\
\hline \multirow{4}{*}{$\begin{array}{l}\text { Pt-fcc } \\
(111)\end{array}$} & fcc & 58.7 & 0.957 \\
\hline & hcp & 56.9 & 0.953 \\
\hline & top & 58.5 & 1.580 \\
\hline & bridge & 57.4 & 1.105 \\
\hline
\end{tabular}

corrections due to the vibrational motion of the adsorbed atoms. We expect this correction to be approximately the same for fcc and hcp sites on the same metal and somewhat larger for top sites. For all closest packed (fcc and hcp) metals, we calculate that adsorption at the fcc site is favored with respect to the hcp site and, except for $\mathrm{Ru}$, the difference is about $1-2 \mathrm{kcal} / \mathrm{mol}$, indicating that fcc is the preferred site at ML coverage. For Ru the difference between fcc and hcp is 
TABLE II. Hydrogen binding energy and vibrational frequency on metal surfaces at different surface coverages. $D_{e}$ indicates the electronic binding energy in $\mathrm{kcal} / \mathrm{mol}$ [no corrections from zero point vibrational energy (ZPE)]. The frequency $\nu$ refers to the symmetric stretching normal to the surface and is expressed in $\mathrm{cm}^{-1} . D_{0}$ is the ZPE corrected value in $\mathrm{kcal} / \mathrm{mol}$ obtained from $D_{e}$ and $\nu$. Values in parentheses are estimates obtained as described in the text.

\begin{tabular}{|c|c|c|c|c|c|c|c|c|c|c|c|c|c|c|c|}
\hline Coverage & Metal & Site & $D_{e}$ & $\nu$ & $D_{0}$ & Metal & Site & $D_{e}$ & $\nu$ & $D_{0}$ & Metal & Site & $D_{e}$ & $\nu$ & $D_{0}$ \\
\hline 1/3 ML & & & 68.1 & 1069 & 64.6 & & & 62.4 & 1235 & 60.6 & & & 62.0 & 1168 & 60.3 \\
\hline 2/3 ML & $\mathrm{Fe}$ & cap & 65.1 & (1101) & $(63.5)$ & Co & fcc & 60.7 & (1276) & (58.9) & $\mathrm{Ni}$ & fcc & 60.6 & (1225) & (58.9) \\
\hline $1 \mathrm{ML}$ & $\mathrm{bcc}$ & & 64.7 & 1133 & 63.1 & hcp & & 60.3 & 1317 & 58.4 & fcc & & 60.1 & 1282 & 58.3 \\
\hline 4/3 ML & & cap, cap & 40.1 & $\cdots$ & $(38.5)$ & & fcc, hcp & 35.3 & $\cdots$ & $(33.4)$ & & fcc, hcp & 36.3 & $\cdots$ & (34.5) \\
\hline 1/3 ML & & & 67.8 & 1237 & 66.0 & & & 62.5 & 1184 & 60.8 & & & & & \\
\hline $2 / 3 \mathrm{ML}$ & $\mathrm{Fe}$ & fcc & 66.8 & (1216) & $(65.1)$ & Co & fcc & 61.3 & (1252) & $(59.5)$ & & & & & \\
\hline $1 \mathrm{ML}$ & fcc & & 66.6 & 1196 & 64.9 & fcc & & 60.7 & 1320 & 58.8 & & & & & \\
\hline 4/3 ML & & fcc, hcp & 39.9 & $\cdots$ & $(38.2)$ & & fcc, hcp & 36.0 & $\cdots$ & (34.1) & & & & & \\
\hline $1 / 3 \mathrm{ML}$ & & & 62.6 & 1154 & 61.0 & & & & & & & & & & \\
\hline 2/3 ML & & hcp & 61.3 & (1202) & (59.6) & & & & & & & & & & \\
\hline $1 \mathrm{ML}$ & & & 60.7 & 1251 & 58.9 & & & & & & & & & & \\
\hline 1/3 ML & $\mathrm{Ru}$ & & 63.4 & 1119 & 61.8 & & & 59.5 & 1151 & 57.9 & & & 60.2 & 1146 & 58.6 \\
\hline 2/3 ML & hcp & fcc & 61.7 & (1127) & $(60.1)$ & $\mathrm{Rh}$ & fcc & 58.9 & (1229) & $(57.1)$ & $\mathrm{Pd}$ & fcc & 59.4 & (1090) & (57.8) \\
\hline $1 \mathrm{ML}$ & & & 60.5 & 1135 & 58.9 & fcc & & 58.5 & 1308 & 56.6 & fcc & & 57.5 & 1035 & 56.0 \\
\hline 4/3 ML & & fcc, hcp & 36.2 & $\cdots$ & $(34.6)$ & & fcc, hcp & 37.6 & $\cdots$ & $(35.7)$ & & fcc, hcp & 34.7 & $\cdots$ & $(33.2)$ \\
\hline 1/3 ML & & & & & & & & 63.0 & 2345 & 59.7 & & & & & \\
\hline 2/3 ML & & & & & & & top & 62.4 & (2339) & $(59.1)$ & & & & & \\
\hline $1 \mathrm{ML}$ & & & & & & & & 62.0 & 2333 & 58.7 & & & & & \\
\hline 1/3 ML & & & 64.9 & 1177 & 63.2 & $\mathrm{Ir}$ & & 61.5 & 1244 & 59.7 & & & 59.9 & 1155 & 58.3 \\
\hline 2/3 ML & Os & fcc & 62.7 & (1183) & $(61.0)$ & fcc & fcc & 60.8 & (1267) & $(59.0)$ & $\mathrm{Pt}$ & fcc & 59.3 & (1164) & (57.6) \\
\hline $1 \mathrm{ML}$ & hcp & & 61.4 & 1189 & 59.7 & & & 60.1 & 1290 & 58.3 & fcc & & 58.4 & 1173 & 56.7 \\
\hline 4/3 ML & & top, fcc & 53.1 & $\ldots$ & $(46.6)$ & & top, fcc & 56.2 & $\ldots$ & $(54.4)$ & & top, fcc & 49.2 & $\ldots$ & (45.9) \\
\hline
\end{tabular}

only $0.1 \mathrm{kcal} / \mathrm{mol}$ so that ZPE corrections would be needed to determine which of the two sites is more stable. Except for third row metals, the top position is considerably less favorable than either fcc or hcp. For Ir, we find that the top position is $1.4 \mathrm{kcal} / \mathrm{mol}$ more stable than fcc. We expect the ZPE correction to be larger for top adsorption positions than for either fcc or hcp so we considered both adsorption geometries for further investigations on the larger SUC.

Our conclusion is that fcc geometry is favored on all metals with the possible exceptions of $\mathrm{Ru}$, where hcp is equally favored, and Ir, where top may be equally favored.

Based on experimental results for several metals and on previous calculations, we expect the barrier for surface diffusion of hydrogen to be relatively small. To verify this assumption, we computed the energy for the bridge position between fcc and hcp sites on all the closest packed surfaces considered. In the case of Fe-bcc (110) there are two distinct bridge positions between equivalent cap sites. Although the bridge position is not the exact transition state and despite the fact that we used ML coverage, the energy of the bridge position provides an estimate of the barrier for surface diffusion. We thus estimate this barrier to be around $5 \mathrm{kcal} / \mathrm{mol}$ for $\mathrm{Fe}, \mathrm{Co}$, and $\mathrm{Ni}$, around 3 to $5 \mathrm{kcal} / \mathrm{mol}$ for $\mathrm{Ru}, \mathrm{Rh}$, and $\mathrm{Pd}$, and close to 1 or $2 \mathrm{kcal} / \mathrm{mol}$ for Os, Ir, and Pt. In the case of Ir and Pt, the bridge position has lower energy than the hcp.

We used the $\sqrt{3} \times \sqrt{3} R\left(30^{\circ}\right)$ SUC to calculate the coverage dependence of the $\mathrm{BE}$ for the most stable positions. This is suitable for $1 / 3,2 / 3$, and $3 / 3 \mathrm{ML}$ coverages and for coverages past $1 \mathrm{ML}$. Although on certain metal surfaces, most notably $\mathrm{Ni}(111)$ (Ref. 15) and $\mathrm{Fe}(110)$ (Refs. 15 and 16) there is evidence of adsorption at different sites for cov- erages near $1 / 2 \mathrm{ML}$ at low temperature, probably due to an overall minimization of adsorbates interaction, we found that for the coverages of $1 / 3$ and $2 / 3$ ML the most stable adsorption geometry comprises pure fcc sites. A more detailed description of the hydrogen behavior at various coverages and on specific metals is beyond the scope of this article. We refer the interested reader to the literature regarding specific metal surfaces. ${ }^{15}$ The computed BEs are reported in Table II. We find that for all systems there is a gradual decrease in the BE going from $1 / 3 \mathrm{ML}$ to $1 \mathrm{ML}$ by an average of $2.0 \mathrm{kcal} /$ mol out of 61.6 or $3.2 \%$. The largest changes $(\approx 3 \mathrm{kcal} / \mathrm{mol})$ were for $\mathrm{Os}, \mathrm{Pd}$, and $\mathrm{Ru}$. The smallest changes $(\approx 1 \mathrm{kcal} /$ mol) were for Ir on-top, Rh, and Fe fcc.

To investigate coverage past $1 \mathrm{ML}$, we studied all plausible adsorption configurations corresponding to 4/3 ML for the third row metals. We report in Table II the most stable configuration. For first and second row elements we only considered adsorption in the cap positions ( $1 \mathrm{ML}$ fcc and $1 / 3$ ML hcp). Going from $1 \mathrm{ML}$ to $4 / 3 \mathrm{ML}$ coverage leads to a large drop (10-30 kcal/mol) in binding energy for all systems except Ir. For Ir, the binding energy decreases only by $5.8 \mathrm{kcal} / \mathrm{mol}$. Since the $\mathrm{H}-\mathrm{H}$ bond strength is $110 \mathrm{kcal} / \mathrm{mol}$, a bond strength of less than $55 \mathrm{kcal} / \mathrm{mol}$ will lead to a state unstable with respect to desorption. With the possible exception of Ir, the binding energy for 4/3 ML is generally too low to allow a significant population of this state in equilibrium with gas phase $\mathrm{H}_{2}$ even under very drastic conditions. A more detailed discussion of adsorption on $\mathrm{Ir}$ is reported in Sec. IV.

In order to compare our binding energies $D_{e}$ to experimental data, we corrected for the ZPE vibrational mode perpendicular to the surface. We computed the harmonic fre- 
TABLE III. Comparison of computed and experimental data. Values for $D_{0}$ are reported for $1 / 3 \mathrm{ML}$ coverage to compare with experimental values at low coverage. The range of computed frequencies is given by the 1/3 ML and $1 \mathrm{ML}$ values. The last column contains the references used for the experimental results.

\begin{tabular}{|c|c|c|c|c|c|}
\hline \multirow[b]{2}{*}{ Surface } & \multicolumn{2}{|c|}{$D_{0}(\mathrm{kcal} / \mathrm{mol})$} & \multicolumn{2}{|c|}{$\omega\left(\mathrm{cm}^{-1}\right)$} & \multirow[b]{2}{*}{ References } \\
\hline & Comp. & Expt. & Comp. & Expt. $^{\mathrm{a}}$ & \\
\hline Fe-bcc (110) & 64.6 & 64.8 & $1069-1133$ & 1060 & 3 \\
\hline Co-hcp (001) & 60.6 & 59.8 & $1235-1317$ & & 3 \\
\hline Ni-fcc (111) & 60.3 & 63.1 & $1168-1282$ & 1170 & 3,17 \\
\hline Ru-hep (001) & 61.8 & $61.2-66.0$ & $1119-1135$ & $1112-1136$ & 3 \\
\hline Rh-fcc (111) & 57.9 & 60.9 & $1151-1308$ & $1088-1100$ & 3,18 \\
\hline Pd-fcc (111) & 58.6 & 62.1 & $1035-1146$ & 998 & 3 \\
\hline Ir-fcc (111) & 59.7 & 58.1 & $2333-2345$ & 2030 & 3,19 \\
\hline Pt-fcc (111) & 58.3 & $56.6-60.9$ & $1155-1173$ & 1230 & 3 \\
\hline
\end{tabular}

${ }^{a}$ Most experimental measurements are based on HREELS with typical accuracy of $8 \mathrm{meV}=65 \mathrm{~cm}^{-1}$.

quencies for this vibrational mode for $1 / 3$ and $3 / 3 \mathrm{ML}$ coverages using numerical differentiation. The frequencies are reported in Table II. The frequency for $2 / 3 \mathrm{ML}$ coverage was estimated as the average of the $1 / 3$ and $3 / 3 \mathrm{ML}$ values. These frequencies were used to correct for vibrational zero point energy for coverages up to $1 \mathrm{ML}$, leading to the $D_{0} \mathrm{BE}$ in the last column of Table II. The ZPE at 4/3 ML was estimated by assuming that the correction for hcp sites is the same as for fcc sites at $1 \mathrm{ML}$ and the correction for all top sites is the same as computed for the top position on Ir at 1 ML coverage. The $D_{0}$ values are the finite-difference BEs at $0 \mathrm{~K}$, i.e., the energies gained by adding one third of a monolayer and represent an estimate to the differential BEs measured experimentally. As the diffusion barriers are low, the motion parallel to the surface is expected to give negligible contributions to the ZPE and to be highly anharmonic. This motion is included in the statistical treatment reported later in this article but it is neglected in the present comparison of low temperature binding energies.

Our results refer to unrelaxed defect-free surfaces. We investigated the effect of surface relaxation in the case of $\mathrm{Pt}$ by relaxing the first two metal layers. In this case, the hydrogen binding energy increases by $1.2,0.3$, and $0.7 \mathrm{kcal} / \mathrm{mol}$ for $1 / 3,2 / 3$, and $3 / 3 \mathrm{ML}$ coverages, respectively. The average increase of $0.7 \mathrm{kcal} / \mathrm{mol}$ is comparable with the expected accuracy of the computational method.

\section{DISCUSSION}

\section{A. Comparison of binding energies and vibrational frequencies with experiment}

The binding energies $D_{0}$ and symmetric vibrational frequencies $\nu$ reported in Table II are compared with published experimental values in Table III.

Since most experimental BEs refer to low coverage extrapolations, we compare them with the computed values for 1/3 ML. Experimental frequencies are typically measured near ML coverage. The values we compute for ML coverage, however, neglect the coupling between vibrational motion of neighboring atoms and is thus somewhat approximate. Fortunately, the computed coverage dependent frequency shift is small and even large relative errors in the frequencies will have small effects on thermodynamics predictions. To compare with the experimental frequencies we report in Table III the computed values for both 1/3 ML and $1 \mathrm{ML}$, as an estimate of the computational range.

The uncertainty in the experimental binding energies seems to be $4-5 \mathrm{kcal} / \mathrm{mol}$ based on the results reported for $\mathrm{Pt}$ and $\mathrm{Ru}$. For these systems, the calculated values are within the range of experimental results. For the other six systems (we found no experimental results for Os), the mean average discrepancy is $2.0 \mathrm{kcal} / \mathrm{mol}$.

The computed frequencies are within experimental uncertainty of experiment for $\mathrm{Fe}, \mathrm{Ni}$, and $\mathrm{Ru}$, high by $5 \%$ for $\mathrm{Rh}$ and $\mathrm{Pd}$, low by $6 \%$ for $\mathrm{Pt}$, and high by $16 \%$ for Ir (on top).

\section{B. Comparison to previous calculations}

Several detailed computational investigations have been published focusing on one or a few particular metal surfaces. Since it is beyond the scope of this article to provide a complete review of published results, we report only a few of the most recent publications in order to assess the quality of the binding energies we report. We refer the reader to the cited papers for more exhaustive references relative to specific metal surfaces.

The most systematic calculations of hydrogen chemisorption on metal surfaces are by Nordlander et al., ${ }^{20}$ using effective medium theory. However, this work does not address the binding energy dependence on surface coverage. The results from these studies, reported only in graphical form, range approximately from 62 to $67 \mathrm{kcal} / \mathrm{mol}$ over all nine elements. In comparison, for the same surfaces we find the range 59.9-66.1 kcal $/ \mathrm{mol}$. The largest discrepancies are for $\mathrm{Rh}(59.5$ versus $\approx 63 \mathrm{kcal} / \mathrm{mol})$ and Os $(64.9$ versus $\approx 67$ $\mathrm{kcal} / \mathrm{mol})$, with the other values within $\approx 2 \mathrm{kcal} / \mathrm{mol}$ for each surface considered. The trend predicted by Nordlander et $a l .{ }^{20}$ of decreasing binding energies with increasing atomic number within each row is reproduced by our estimates for 1/3 ML coverage but not for higher coverages.

Hydrogen chemisorption on Fe-bcc(110) was studied by Jiang and $\operatorname{Carter}^{21}$ (JC), who employed seven layers with a $14 \times 14 \times 1$ Monkhorst-Pack grid to perform spin polarized, all electron calculations with relaxed surface. We obtain the same order for the occupation of surface sites, i.e., we find cap to be the most stable site, followed by the two bridge positions and the top site. JC report two values for BEs obtained with different density functionals and our BEs are bracketed by those values both at low and high coverage, i.e., fall within the range $65.3-69.2 \mathrm{kcal} / \mathrm{mol}$ for $1 / 4 \mathrm{ML}$ and 63.6-67.1 kcal/mol for $1 \mathrm{ML}$. Our computed bond distances to the surface for ML coverage are $0.04-0.08 \AA$ longer than those reported by JC.

Our computed BEs on Ni appear a few $\mathrm{kcal} / \mathrm{mol}$ smaller than those reported by Greeley and Mavrikakis ${ }^{22}$ (GM) and Kresse and Hafner ${ }^{23}(\mathrm{KH})$. In fact, both GM and $\mathrm{KH}$ report values for $D_{e}$ of 66.6 and $66.0(\mathrm{GM})$ or $65.9(\mathrm{KH}) \mathrm{kcal} / \mathrm{mol}$ for $1 / 4 \mathrm{ML}$ and $1 \mathrm{ML}$, respectively, versus our values of 62.0 and $60.9 \mathrm{kcal} / \mathrm{mol}$. Although our neglect of surface relaxation and spin polarization may contribute to this difference, 
$\mathrm{KH}$ estimate these effects at approximately $2-3 \mathrm{kcal} / \mathrm{mol}$. The difference between our results and those reported by GM and $\mathrm{KH}$ appears to be due mainly to the choice of different pseudopotentials. As reported in Sec. II, the monolayer BE computed with ultrasoft potentials is 67.8 (without spin polarization) or $66.2 \mathrm{kcal} / \mathrm{mol}$ (with spin polarization), in good agreement with GM and $\mathrm{KH}$. As the experimental $\mathrm{BE}$ is reported at $63.1 \mathrm{kcal} / \mathrm{mol}^{3}$ and we estimate the ZPE correction of the order of $1.7 \mathrm{kcal} / \mathrm{mol}$, it is not clear which pseudopotential provides a better description of the binding process.

Our computed $\mathrm{BE}$ for $\mathrm{H}$ on $\mathrm{Ru}(001)$ compares well with the results of Ciobîcă et $a l^{24}$ and Norsk $\varnothing v$ and co-workers ${ }^{25}$ who report values for $D_{e}$ in fcc sites at $1 / 4 \mathrm{ML}$ coverage of $53.5 \mathrm{~kJ} / \mathrm{mol}^{2}$ (Ref. 24) and $0.405 \mathrm{eV}$ (Ref. 25) with respect to gas phase $\mathrm{H}_{2}$. Assuming a $\mathrm{BE}$ for $\mathrm{H}_{2}$ of $4.58 \mathrm{eV}$, as computed by KH with a similar potential, we obtain a $D_{e}$ with respect to atomic $\mathrm{H}$ of 65.6 and $62.1 \mathrm{kcal} / \mathrm{mol}$, respectively, which bracket our $63.4 \mathrm{kcal} / \mathrm{mol}$ computed for $1 / 3 \mathrm{ML}$ coverage. Ciobîcă et al. also found that the energy difference between fcc and hcp sites is extremely small with the fcc slightly more stable.

Hydrogen chemisorption on $\mathrm{Rh}(111)$ was studied with density functional techniques by Mavrikakis et al. ${ }^{26}$ and Lai. ${ }^{27}$ They find BEs $\left(D_{e}\right)$ of 64.3 or $60.4 \mathrm{kcal} / \mathrm{mol},{ }^{26} \mathrm{de}-$ pending on the functional used, at 1/4 ML coverage and 63.0 or $64.9 \mathrm{kcal} / \mathrm{mol},{ }^{27}$ depending on the number of metal layers, for ML coverage. These BEs are slightly higher than ours, with the difference probably due to the use of different pseudopotentials and the use of slabs with a different number of layers.

Dong and $\mathrm{Hafner}^{28}$ report a BE $\left(D_{e}\right)$ on $\mathrm{Pd}(111)$ of 64.3 $\mathrm{kcal} / \mathrm{mol}$ for $2 / 3 \mathrm{ML}$, computed with ultrasoft potential. Pallassana et al. ${ }^{29}$ report $D_{e}=61.3 \mathrm{kcal} / \mathrm{mol}$ at ML coverage for the same surface, using a norm conserving potential. This value increases to $63.8 \mathrm{kcal} / \mathrm{mol}$ for $1 / 3 \mathrm{ML}$ coverage. Once again, the ultrasoft potential appears to provide larger binding energies than the more traditional norm conserving ones.

Mavrikakis and co-workers ${ }^{30}$ report $D_{e}$ for $\mathrm{H}$ on $\operatorname{Ir}(111)$ at $1 / 4 \mathrm{ML}$ coverage in top position of 63.0 or $60.4 \mathrm{kcal} / \mathrm{mol}$, depending on the density functional used. For the fcc site, the BE becomes 60.0 or $56.5 \mathrm{kcal} / \mathrm{mol}$. These values are close to our computed results for $1 / 3 \mathrm{ML}$ coverage.

Chemisorption on $\mathrm{Pt}(111)$ was investigated recently by Nørskov and co-workers ${ }^{25}$ and Légaré ${ }^{31}$ Nørskov et al. report a $\mathrm{BE}$ with respect to gas phase $\mathrm{H}_{2}$ of $0.25 \mathrm{eV} /$ atom at 1/4 ML coverage. Depending on the reference BE for $\mathrm{H}_{2}$, this corresponds to chemisorption energies $D_{e}$ $=60.5 \mathrm{kcal} / \mathrm{mol}$ (using experimental $\mathrm{H}_{2} \mathrm{BE}$ of $4.75 \mathrm{eV}$ ) or $58.6 \mathrm{kcal} / \mathrm{mol}$ [using DFT $\mathrm{H}_{2} \mathrm{BE}$ of $4.58 \mathrm{eV}$ (Ref. 23)]. Légaré reports slightly larger values: $64.1 \mathrm{kcal} / \mathrm{mol}$ at $1 / 4$ ML coverage and $63.9 \mathrm{kcal} / \mathrm{mol}$ at ML coverage. Both sets of results are based on ultrasoft potentials and the difference between them is likely due to the use of different density functionals.

We are not aware of theoretical studies of hydrogen chemisorption on $\mathrm{Co}(001)$ and $\mathrm{Os}(001)$.

Based on the published results summarized above as well as our computations, we conclude that the effect of
TABLE IV. Interpolating functions used to fit the computed binding energies $D_{0}(\theta)$ in $\mathrm{kcal} / \mathrm{mol}$. These are obtained as the least squares fit of a line to the data in Table II.

\begin{tabular}{lll}
\hline \hline Fe-bcc (110) & Co-hcp (001) & Ni-fcc (111) \\
$65.23-2.25 \theta$ & $61.50-3.30 \theta$ & $61.17-3.00 \theta$ \\
Fe-fcc $(111)$ & Co-fcc (111) & \\
$66.43-1.65 \theta$ & $61.70-3.00 \theta$ & \\
Ru-hcp (001) & Rh-fcc (111) & Pd-fcc (111) \\
$63.17-4.35 \theta$ & $58.50-1.95 \theta$ & $60.07-3.90 \theta$ \\
Os-hcp (001) & Ir-fcc (111) & Pt-fcc (111) \\
$64.80-5.25 \theta$ & $60.17-1.50 \theta$ & $59.13-2.40 \theta$ \\
\hline \hline
\end{tabular}

selecting different density functional theory (DFT) functionals within the GGA approximation and different ECPs may be of as large as $\approx 6 \mathrm{kcal} / \mathrm{mol}$ on the binding energies. By contrast, neglecting spin polarization may introduce errors of $1-2 \mathrm{kcal} / \mathrm{mol}$, similar to the neglect of surface relaxation.

\section{Estimate of equilibrium surface coverage}

We now derive an estimate of equilibrium surface coverage from statistical thermodynamics. Although the following treatment is completely general, in the interest of readability we will provide extensive numerical examples and simulations to illustrate our results only for the case of $\operatorname{Ir}(111)$, limiting applications to other surfaces to coverage estimates (Figs. 5 and 6). The more complete treatment applied to Ir can easily be extended to other surfaces from the data in Table II and the expressions provided later in this document.

To estimate the equilibrium surface coverage from statistical thermodynamics, we need to make a few assumptions regarding the nature of the adsorbed hydrogen and the shape of the coverage-dependent binding energy. Some information is available from the literature regarding some of the surfaces considered, however it is likely that some surfaces may require different models. We will develop three simple models for adsorbed hydrogen that describe a variety of limiting behaviors. We find that most features observed experimentally in adsorption profiles and temperature programmed desorption (TPD) spectra can be reproduced within these models and that these results are consistent with the available surface science experiments (except for effects connected to phase changes in the adsorbate).

First we need to develop a means to interpolate the calculated binding energies over the range of coverages likely to be of interest. Experimental observations suggest that the shape of the interpolating function is not simple. Thus, for $\mathrm{Pt}$ and Ir, the binding energy is observed to be almost constant between 0.3 and $0.6 \mathrm{ML}$, to increase rapidly in the low coverage region and to decrease sharply approaching $1 \mathrm{ML}^{3}$. Since we cannot capture such behavior by sampling the binding energy only at $1 / 3,2 / 3$, and $3 / 3$ ML coverages and since the computed values appear to change roughly linearly with coverage up to $1 \mathrm{ML}$, we least squares fit the calculated data in Table II to a straight line. The fitting functions are reported in Table IV for all surfaces considered. 


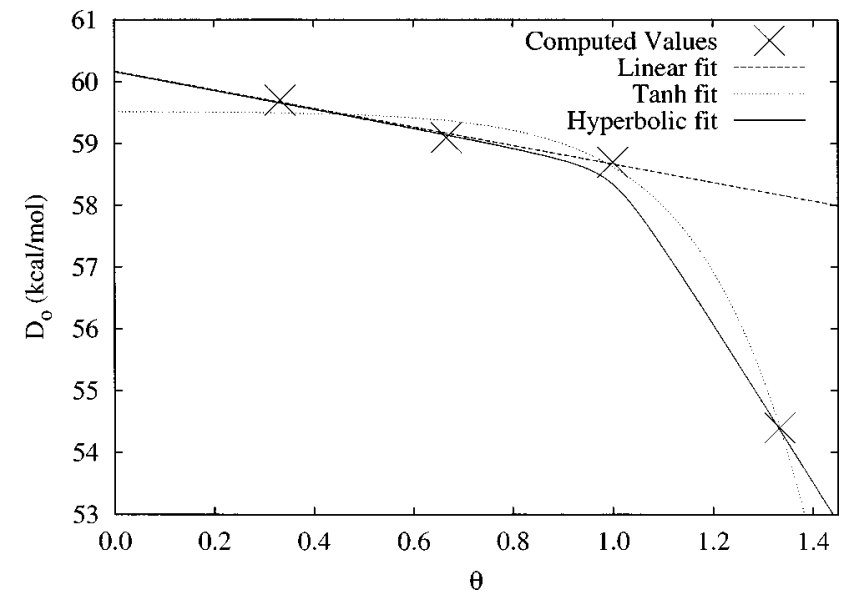

FIG. 1. Fitting functions used to interpolate the computed binding energies $D_{0}$ on $\operatorname{Ir}(111)$.

We now investigate for the case of iridium the effect of fitting these data to other functions and extending the fit past $1 \mathrm{ML}$ coverage. We require that all interpolating functions be continuous and differentiable in the region of interest. For future reference, we refer to the linear fit of points up to 1 ML as the "linear" fit. Of course, we expect this fit to fail for coverages greater than $1 \mathrm{ML}$.

The most stable configuration with four atoms on the $\operatorname{Ir}(111) \sqrt{3} \times \sqrt{3} R\left(30^{\circ}\right)$ SUC has three atoms near top positions and one in fcc. The BE to remove the fourth atom is computed to be $D_{e}=56.2 \mathrm{kcal} / \mathrm{mol}$. Assuming that the fourth atom in fcc position has the same zero point energy as an fcc atom at 1 ML coverage, we estimate the value $D_{0}$ $=54.4 \mathrm{kcal} / \mathrm{mol}$ for $4 / 3 \mathrm{ML}$. We can now fit a smooth function to the values at $1 / 3,2 / 3,3 / 3$, and $4 / 3 \mathrm{ML}$ as shown in Fig. 1. To assess the effect on the results since there are many plausible choices of the fitting function, we selected two rather different forms to determine how the final results are affected by this selection.

Tanh. We used a four parameter hyperbolic tangent least squares fit, namely, $D_{0}(\theta)=30.05+29.47 \tanh (4.809-$ $2.724 \theta$ ). We refer to this function as the "tanh" fit.

Hyperbolic. We used a hyperbole having as asymptotes the lines describing the binding energy up to $1 \mathrm{ML}$, as obtained in the linear fit, and the line connecting the points at $3 / 3$ and 4/3 ML. The hyperbole is defined as the set of points whose distances from the two asymptotes multiplied together equal a given constant $\alpha$. When the equations for the two asymptotes are $a_{1} \theta+b_{1}$ and $a_{2} \theta+b_{2}$, the hyperbole takes the form

$$
\begin{aligned}
D_{0}(\theta)= & \frac{a_{1} \theta+b_{1}+a_{2} \theta+b_{2}}{2} \\
& -\frac{\sqrt{\left(a_{1} \theta+b_{1}-a_{2} \theta-b_{2}\right)^{2}+4 \beta}}{2},
\end{aligned}
$$

where $\beta=\alpha \sqrt{\left(1+a_{1}^{2}\right)\left(1+a_{2}^{2}\right)}$. In our particular case, the values used are (in $\mathrm{kcal} / \mathrm{mol}$ ) $a_{1}=-1.50, b_{1}=60.17$, $a_{2}=-12.90$, and $b_{2}=71.60$. We refer to this function as the "hyperbolic" fit. By making $\alpha$ small enough we can ap- proach the linear fit in the region below 1 ML with arbitrary precision. For our modeling we chose $\alpha=0.005$.

Figure 1 illustrates the quality of the fit obtained with the three fitting functions considered. We will find that the choice of the fitting function can affect drastically the shape of TPD spectra while leading to only minor changes in the predicted equilibrium coverage.

Several microscopic models have been proposed to mimic hydrogen chemisorption on specific metals, ${ }^{4,32}$ but none of them is universally accepted. In very general terms, the behavior of adsorbed hydrogen must be between the two limiting cases of quantum delocalized two-dimensional gas and covalently bound site-anchored hydride. Simulations are further complicated by the presence of additional adsorbate phases depending on temperature, coverage, and which surface is being considered.

To provide a simple and intuitive description of the system, we considered three possible models for adsorbed hydrogen.

Ideal surface gas. In this model we regard the hydrogen as an ideal surface gas. Since the only interatomic interactions are through the coverage dependence of the binding energy, we expect this approach to be correct only in the low coverage and high temperature limit.

Hard-disk surface gas. This model includes the exclusion part of the intermolecular interactions. This describes saturation effects and is expected to describe correctly the behavior of most surfaces at high temperature.

Anchored. In this model each hydrogen atom is considered to be anchored to a specific surface site. This should describe the low temperature limit for surfaces having high barriers for surface diffusion.

\section{Gas phase treatment}

The critical parameters for gas phase hydrogen $\left(\mathrm{H}_{2}\right)$ are $T_{c}=32.97 \mathrm{~K}$ and $P_{c}=12.76 \mathrm{~atm}$. Based on the principle of corresponding states, we expect $\mathrm{H}_{2}$ to behave essentially like an ideal gas for temperatures greater than $2 T_{c}(\approx 66 \mathrm{~K})$ and up to $5 P_{c}(\approx 64 \mathrm{~atm})$. For higher pressures, deviation from ideal behavior are expected, depending on the temperature. For the scope of this study we will consider $\mathrm{H}_{2}$ as an ideal gas.

The canonical partition function $Q_{g}$ for $N_{g}$ gas phase hydrogen molecules is

$$
Q_{g}=\frac{q_{g}^{N_{g}}}{N_{g} !} .
$$

The molecular partition function $q_{g}$ includes the ZPE in the reference energy for the gas phase molecule,

$$
q_{g}=V \frac{\left(2 \pi m_{\mathrm{H}_{2}} k_{B} T\right)^{3 / 2}}{h^{3}} \frac{1}{1-e^{-h \nu / k_{B} T}} \frac{8 \pi^{2} I k_{B} T}{2 h^{2}}
$$

where $\nu$ is the vibrational frequency, $I$ the moment of inertia, $m_{\mathrm{H}_{2}}$ the molecular mass, $T$ the temperature, and $k_{B}$ and $h$ Boltzmann and Plank constants, respectively. The chemical potential for the gas phase is

$$
\mu_{g}=-R T \ln \left(q_{g} / N_{g}\right) \text {. }
$$


For the computation of the partition function we used an $\mathrm{H}_{2}$ bond distance of $0.7414 \AA$ and a vibrational frequency of $4341 \mathrm{~cm}^{-1}$.

\section{E. Surface treatment}

\section{Formalism}

We adopt the following formalism, common to all the approaches used to describe the adsorbed atoms. $\beta=1 / R T$; ( $R=$ gas constant); $N_{a}$ is the number of adsorbed atoms; $A$ is the surface area; $\rho$ is the surface density $\left(N_{a} / A\right) ; q_{a}$ is the partition function relative to one adsorbed atom; $Q_{a}$ is the partition function relative to $N_{a}$ adsorbed atoms; [ $N_{a}$ ] equals the moles of adsorbed atoms per unit area; $N_{s}$ is the number of surface metal atoms; $\left[N_{s}\right]$ equals the moles of surface metal atoms per unit area; $\nu_{S}$ is the symmetric stretching frequency; $q_{v}$ is $\left\{\left[1-\exp \left(h \nu_{s} / k_{B} T\right)\right]^{-1}\right\}$, the adsorbed atom vibrational partition function; $\theta$ is the surface coverage $\left\{\left[N_{a}\right] /\left[N_{s}\right]=N_{a} / N_{s}\right\} ; \theta_{M}$ is the maximum possible value of $\theta ; E(\theta)$ is the adsorption energy relative to gas phase $\mathrm{H}_{2}$ including zero point energy. $E(\theta)>0$ when two adsorbed atoms are more stable than one gas phase molecule at $0 \mathrm{~K}$. $E(\theta)$ refers to $1 \mathrm{~mol}$ of adsorbed atoms; $F(\theta)$ is $[E(\theta)$ $+\theta d E(\theta) / d \theta] ; \mu_{a}$ is the chemical potential of the adsorbed atoms; and $\Lambda$ is the thermal De Broglie wavelength for hydrogen $\left[\left(h^{2} / 2 \pi m_{\mathrm{H}} k_{B} T\right)^{1 / 2}\right]$.

\section{Quantum correction}

We are interested in temperatures between $100 \mathrm{~K}$, typical of surface science studies, and $800 \mathrm{~K}$, typical of industrial catalysis, and in all possible values of surface coverage $\theta$. The treatment is complicated by the fact that for an ideal Fermi-Dirac two dimensional (2D) gas at $100 \mathrm{~K}$ and near monolayer coverage (surface density of 0.15 molecules/ $/ \AA^{2}$ ) the quantum correction to the classical behavior (see derivation below) accounts for about $30 \%$ of the chemical potential. Although this correction drops to $4 \%$ at $300 \mathrm{~K}$ and $1 \mathrm{ML}$ or at $100 \mathrm{~K}$ and $1 / 3 \mathrm{ML}$, it must be included in the statistical treatment for quantitative results to be meaningful.

The treatment of a general 2D Fermi-Dirac gas requires a number of approximations and numerical summations that would obscure the physical meaning of the resulting formulas. Hence we chose to adopt the following ad hoc approximate approach. We assume that the quantum correction for all the adsorption models considered here is the same as for an ideal 2D gas at the same density and temperature. This correction is added to the free energy obtained from the classical partition function. This approximation is consistent with the binding energies reported in the preceding section, which refer to a classical description of the hydrogen nuclei. This makes it questionable to correct for quantum behavior in the binding energies.

In order to proceed, we must compute the statistical free energy for an ideal 2D fermion gas in both the weakly degenerate and the classical limits.

Classical limit. We are interested in surface coverage due to the reaction

$$
\mathrm{H}_{2(g)} \rightleftharpoons 2 \mathrm{H}_{a},
$$

where $\mathrm{H}_{a}$ represents adsorbed atoms. The canonical partition function for $N_{a}$ adsorbed atoms is

$$
Q_{a}=\frac{q_{a}^{N_{a}}}{N_{a} !} e^{\beta N_{a} E} .
$$

Since the gas is ideal, we consider constant binding energy: $E(\theta)=E$. The atomic partition function $q_{a}$ contains two translational degrees of freedom,

$$
q_{a}=A \frac{2 \pi m_{\mathrm{H}} k_{B} T}{h^{2}}=A / \Lambda^{2} .
$$

The chemical potential in the classical limit is

$$
\mu_{a}^{c l}=-R T\left(\frac{\partial \ln Q_{a}}{\partial N_{a}}\right)_{A, T}=R T \ln \left(\rho \Lambda^{2}\right)-E .
$$

Weakly degenerate limit. The grand canonical partition function is

$$
\Xi_{a}=\prod_{k}\left(1+\lambda e^{-\epsilon_{k} /\left(k_{B} T\right)+\beta E}\right),
$$

where $\lambda=e^{\beta \mu_{a}}$, the product is over all quantum states $k$, and $\epsilon_{k}$ indicates the translational energy of state $k$ with quantum numbers $n_{x}$ and $n_{y}$ :

$$
\epsilon_{k}=\frac{h^{2}}{8 m_{\mathrm{H}} A}\left(n_{x}^{2}+n_{y}^{2}\right) \text {. }
$$

Once again we consider constant binding energy $E(\theta)=E$. The number of particles is obtained as

$$
N_{a}=k_{B} T\left(\frac{\partial \ln \Xi_{a}}{\partial \mu_{a}}\right)=\sum_{k} \frac{\lambda e^{-\epsilon_{k} /\left(k_{B} T\right)+\beta E}}{1+\lambda e^{-\epsilon_{k} /\left(k_{B} T\right)+\beta E}} .
$$

Notice that Eq. (8) is correct only for constant $E$. For large areas $A$, we approximate the sum with an integral over the energy by introducing the density of states for a $2 \mathrm{D}$ perfect gas $\omega(\epsilon)=2 \pi m_{\mathrm{H}} A / h^{2}$ :

$$
\begin{aligned}
N_{a} & =\frac{2 \pi m_{\mathrm{H}} A}{h^{2}} \int_{0}^{\infty} \frac{\lambda e^{-\epsilon /\left(k_{B} T\right)+\beta E}}{1+\lambda e^{-\epsilon /\left(k_{B} T\right)+\beta E}} d \epsilon \\
& =\frac{A}{\Lambda^{2}} \ln \left(1+\lambda e^{\beta E}\right),
\end{aligned}
$$

which can be solved for $\lambda=\exp \left(\beta \mu_{a}\right)$ and yields finally

$$
\mu_{a}=-E+R T \ln \left(e^{\rho \Lambda^{2}}-1\right) .
$$

The classical limit Eq. (5) is recovered by expanding the exponential and considering small values of $\rho \Lambda^{2}$ :

$$
\begin{aligned}
\mu_{a} & =-E+R T \ln \left[\left(\rho \Lambda^{2}\right)+\frac{\left(\rho \Lambda^{2}\right)^{2}}{2 !}+\frac{\left(\rho \Lambda^{2}\right)^{3}}{3 !}+\cdots\right] \\
& \approx-E+R T \ln \left(\rho \Lambda^{2}\right) .
\end{aligned}
$$

The quantum correction is thus

$$
\text { Quantum correction }=\mu_{a}-\mu_{a}^{c l}=R T \ln \left(\frac{e^{\rho \Lambda^{2}}-1}{\rho \Lambda^{2}}\right) \text {. }
$$




\section{Ideal surface gas model}

Since the barrier for translation parallel to the surface is generally low, we consider the case of an ideal twodimensional surface gas. That is, we assume that adsorbed hydrogen can diffuse parallel to the surface without a barrier and that the only interatomic interactions are through the $\theta$ dependency of the binding energy. We include contributions from vibrations normal to the surface explicitly. We expect this model to fail for high values of $\theta$, when detailed interatomic interactions become more important.

The canonical partition function is

$$
Q_{a}=\frac{q_{a}^{N_{a}}}{N_{a} !} e^{\beta N_{a} E(\theta)},
$$

where the classical atomic partition function $q_{a}$ is

$$
q_{a}=A \frac{2 \pi m_{\mathrm{H}} k_{B} T}{h^{2}} \frac{1}{1-e^{-h v_{s} / k_{B} T}}=\frac{A q_{v}}{\Lambda^{2}} .
$$

Accordingly, the classical chemical potential is

$$
\begin{aligned}
\mu_{a}^{c l} & =-R T\left(\frac{\partial \ln Q_{a}}{\partial N_{a}}\right)_{A, T} \\
& =R T \ln \left(\frac{\rho \Lambda^{2}}{q_{v}}\right)-E(\theta)-\theta \frac{d E(\theta)}{d \theta} \\
& =R T \ln \left(\frac{\rho \Lambda^{2}}{q_{v}}\right)-F(\theta) .
\end{aligned}
$$

Applying the quantum correction from Eq. (12), we obtain

$$
\mu_{a}=R T \ln \left(\frac{e^{\rho \Lambda^{2}}-1}{q_{v}}\right)-F(\theta) .
$$

The equilibrium condition obtained by equating the chemical potentials $\left(\mu_{g}=2 \mu_{a}\right)$ is

$$
\frac{N_{g}}{V}=\left(\frac{q_{g}}{V}\right)\left(\frac{e^{\rho \Lambda^{2}}-1}{q_{v}}\right)^{2} e^{-2 \beta F(\theta)},
$$

and, since $\rho=\theta\left[N_{s}\right]$,

$$
\frac{N_{g}}{V}=\left(\frac{q_{g}}{V}\right)\left(\frac{e^{\theta \Lambda^{2}\left[N_{s}\right]}-1}{q_{v}}\right)^{2} e^{-2 \beta F(\theta)} .
$$

This result shows that it is not possible to define a simple $(\theta$ independent) equilibrium constant for the process. Since $\mathrm{H}_{2}$ is ideal, the equilibrium pressure is

$$
P_{\mathrm{H}_{2}}^{e q}=\frac{k_{B} T N_{g}}{V}=k_{B} T\left(\frac{q_{g}}{V}\right)\left(\frac{e^{\theta \Lambda^{2}\left[N_{s}\right]}-1}{q_{v}}\right)^{2} e^{-2 \beta F(\theta)} .
$$

The predicted surface coverage at any given temperature and pressure can be obtained by solving the equilibrium condition (19). However, this model contains no upper bound to the surface coverage, so that the predicted values of $\theta$ are meaningless when outside the range used to fit the $\theta$ dependence of the binding energy. This shortcoming can easily be fixed by using a fitting function that describes the sharp decrease in binding energy past monolayer coverage. This is illustrated for the case of Ir in Figs. 2(a), 2(b), and 2(c) that reports the predicted values of $\theta$ for temperatures between
100 and $800 \mathrm{~K}$ and selected values of pressure. Figure 2(a) contains the prediction using the linear fit from Table IV while Figs. 2(b) and 2(c) were obtained with the tanh and hyperbolic fits, respectively.

\section{Hard-disk gas model}

It is clear that near saturation conditions the surface hydrogen cannot be regarded as an ideal 2D gas. Since we are interested in both the low coverage and high coverage regimes, we must account for nonideal behavior of adsorbed hydrogen.

Following the approach of Van der Waals, the first correction we introduce is to assign an area to each adsorbed hydrogen. The treatment then becomes identical to the one in the preceding section for the atomic contributions to the partition function with the only difference being that the area available to each molecule depends on surface coverage. The atomic partition function $q_{a}$ is thus

$$
q_{a}=\frac{\left(A-b N_{a}\right) q_{v}}{\Lambda^{2}},
$$

where $b$ is a parameter corresponding to the area occupied by a surface atom. To estimate $b$ we consider that for a saturation coverage $\theta_{M}$ there must be no space left on the surface for further adsorption. This leads to $b=A /\left(N_{s} \theta_{M}\right)$ and hence that $b \rho=\theta / \theta_{M}$.

The surface canonical partition function in the classical limit is

$$
Q_{a}=\frac{q_{a}^{N_{a}}}{N_{a} !} e^{N_{a} E(\theta) / R T},
$$

from which one obtains the chemical potential in the classical limit

$$
\begin{aligned}
\mu_{a}^{c l}= & -R T \ln \frac{q_{a}}{N_{a}}+R T \frac{\theta / \theta_{M}}{1-\theta / \theta_{M}}-F(\theta) \\
= & R T \ln \left(\frac{\rho \Lambda^{2}}{q_{v}}\right)-R T \ln \left(1-\theta / \theta_{M}\right) \\
& +R T \frac{\theta / \theta_{M}}{1-\theta / \theta_{M}}-F(\theta) .
\end{aligned}
$$

Applying the quantum correction from Eq. (12), we obtain the chemical potential

$$
\begin{aligned}
\mu_{a}= & R T \ln \left(\frac{e^{\rho \Lambda^{2}}-1}{q_{v}}\right)-R T \ln \left(1-\theta / \theta_{M}\right) \\
& +R T \frac{\theta / \theta_{M}}{1-\theta / \theta_{M}}-F(\theta) .
\end{aligned}
$$

The equilibrium condition $\left(\mu_{g}=2 \mu_{a}\right)$ yields:

$$
\frac{N_{g}}{V}=\left(\frac{q_{g}}{V}\right)\left(\frac{e^{\rho \Lambda^{2}}-1}{q_{v}}\right)^{2}\left(\frac{e^{\theta /\left(\theta_{M}-\theta\right)}}{1-\theta / \theta_{M}}\right)^{2} e^{-2 \beta F(\theta)} .
$$

The equilibrium pressure is then 
(A) Ideal gas - Linear fit

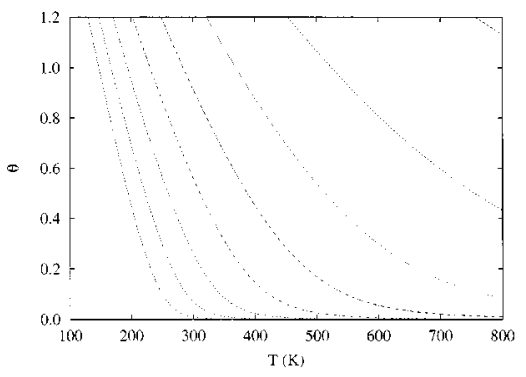

(B) Ideal gas - Tanh fit

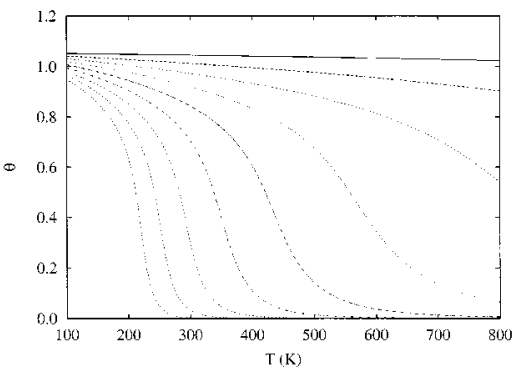

(C) Ideal gas - Hyperbolic fit

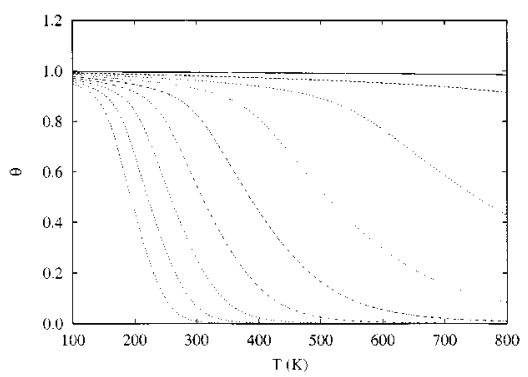

(D) Hard Disk gas - Linear fit

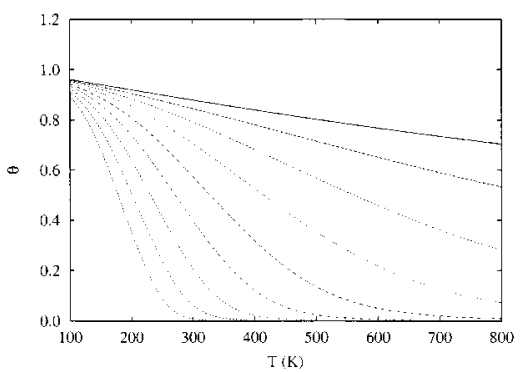

(E) Hard Disk gas - Tanh fit

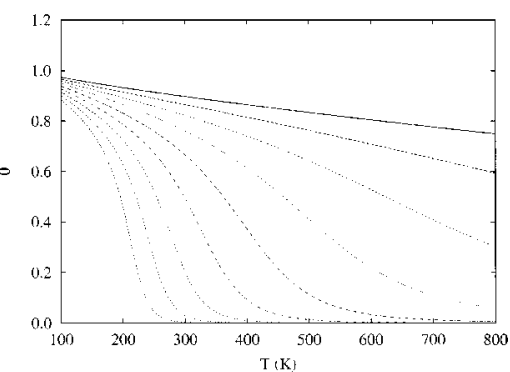

(F) Hard Disk gas - Hyperbolic fit

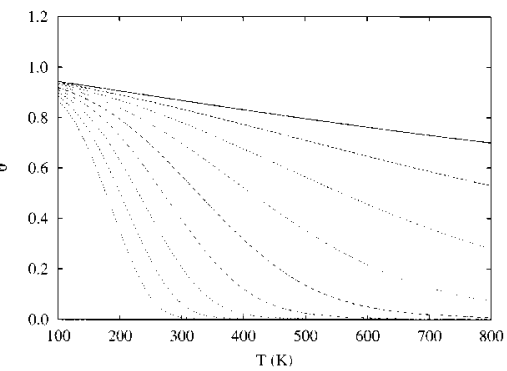

(G) Anchored - Linear fit

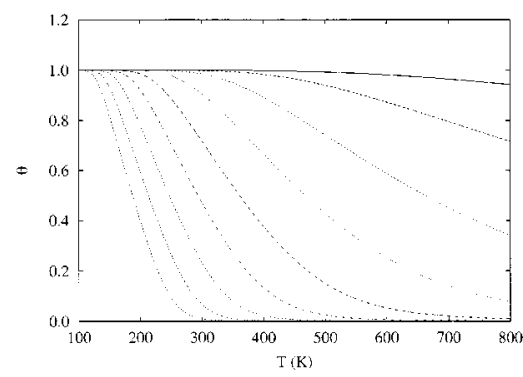

(H) Anchored - Tanh fit

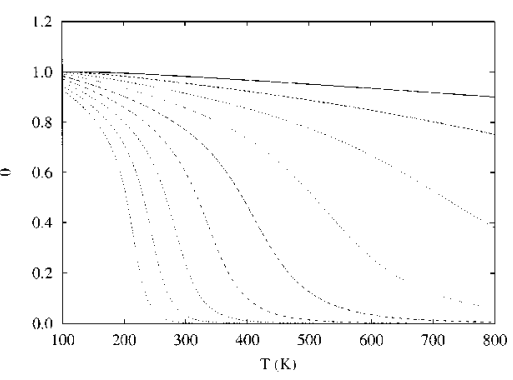

(I) Anchored - Hyperbolic fit

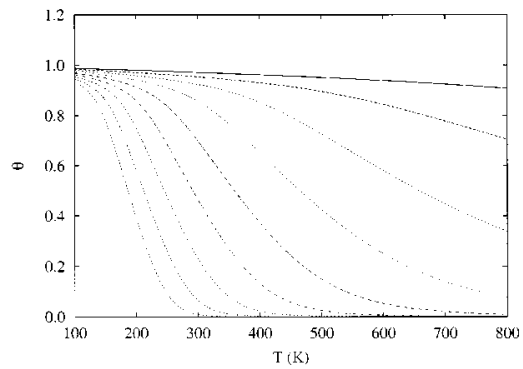

FIG. 2. Predicted equilibrium $\operatorname{Ir}(111)$ surface coverage $\theta$ as a function of temperature using various fits to our calculated energetics with various models. Each line corresponds to one of nine selected values of pressure: $10^{-14}$ (lower, leftmost line) $10^{-12}, 10^{-10}, 10^{-8}, 10^{-6}, 10^{-4}, 10^{-2}, 10^{0}$, and $10^{2}$ atm (upper, rightmost line). All levels of approximation yield qualitatively correct coverages except for the ideal gas model using the linear interpolating function. Independent of the interpolating functions, all models behave like the ideal gas for coverages up to $\approx 0.2$. We expect that real systems will exhibit behavior between the hard-disk model and the anchored model, depending on the specific system and conditions used. 


$$
\begin{aligned}
P_{\mathrm{H}_{2}}^{e q} & =k_{B} T\left(\frac{q_{g}}{V}\right)\left(\frac{e^{\rho \Lambda^{2}}-1}{q_{v}}\right)^{2}\left(\frac{e^{\theta /\left(\theta_{M}-\theta\right)}}{1-\theta / \theta_{M}}\right)^{2} e^{-2 \beta F(\theta)} \\
& =P_{i d} \frac{e^{\theta /\left(\theta_{M}-\theta\right)}}{\left(1-\theta / \theta_{M}\right)^{2}},
\end{aligned}
$$

where $P_{i d}$ is the equilibrium pressure for the ideal surface gas from Eq. (19). In the special case $\theta_{M}=1$, the simpler form is obtained:

$$
P_{\mathrm{H}_{2}}^{e q}=P_{i d}\left[\frac{e^{\theta /(1-\theta)}}{1-\theta}\right]^{2}
$$

To determine $\theta_{M}$, one obvious choice is to assume that hydrogen cannot adsorb past a monolayer coverage leading to $\theta_{M}=1$. This choice is consistent with the use of a linear fitting function for the binding energy. In case the fitting function has a sharp decrease in binding energy near monolayer coverage it is possible to use other values of $\theta_{M}$. In particular, $\theta_{M}$ can be estimated as the value for which $F(\theta)=0$, i.e., the value for which an incoming $\mathrm{H}_{2}$ molecule would experience only a repulsive potential. In the case of Ir with the tanh fit, for instance, one finds $\theta_{M}=1.0533$.

The predicted surface coverage at any given temperature and pressure is obtained by solving equilibrium condition (24). We report numerical solutions for the $\operatorname{Ir}(111)$ surface in Figs. 2(d), 2(e), and 2(f) for selected values of temperature and pressure and with the three choices of fitting function. The behavior at low coverages is similar to the one predicted with the ideal surface gas model.

\section{Anchored hydrogen model}

We now consider the case in which each adsorbed atom is anchored to a specific surface site. We assume there is one available site per surface metal atom and that the adsorbed atom is constrained to a $2 \mathrm{D}$ box of area $A / N_{s} \theta_{M}$ around the site. This corresponds to the limiting case when hydrogen atoms do not migrate on the surface even at low coverage and high temperature.

The surface partition function is

$$
Q_{a}=\frac{q_{a}^{N_{a}}}{N_{a} !\left(N_{a} \theta_{M}-N_{a}\right) !} e^{N_{a} E(\theta) / R T} .
$$

In this case the atomic partition function is

$$
q_{a}=\frac{A q_{v}}{N_{s} \theta_{M} \Lambda^{2}}=\frac{\theta q_{v}}{\theta_{M} \rho \Lambda^{2}},
$$

and the chemical potential in the classical limit is

$$
\mu_{a}^{c l}=R T \ln \left(\frac{\rho \Lambda^{2}}{q_{v}}\right)+R T \ln \left(\frac{1}{1-\theta / \theta_{M}}\right)-F(\theta) .
$$

Applying the quantum correction we obtain

$$
\mu_{a}=R T \ln \left(\frac{e^{\rho \Lambda^{2}}-1}{q_{v}}\right)+R T \ln \left(\frac{1}{1-\theta / \theta_{M}}\right)-F(\theta),
$$

with the equilibrium condition $\left(\mu_{g}=2 \mu_{a}\right)$ expressed as
TABLE V. Summary of main assumptions and conclusions of the three models considered. The value $\theta_{M}=1$ is assumed in order to ease the comparison. $P_{i d}=k_{B} T\left(q_{g} / V\right)\left[\left(e^{\rho \Lambda^{2}}-1\right) / q_{v}\right]^{2} e^{-2 \beta F(\theta)}$.

\begin{tabular}{llll}
\hline \hline & \multicolumn{1}{c}{ Ideal gas } & Hard-disk gas & Anchored hydrogen \\
\hline Comments & $\begin{array}{l}\text { Valid in the high } \\
\text { temperature, low } \\
\text { barrier, low coverage } \\
\text { limit }\end{array}$ & $\begin{array}{l}\text { Valid in the high } \\
\text { temperature, low } \\
\text { barrier limit }\end{array}$ & $\begin{array}{l}\text { Valid in the low } \\
\text { barrier limit }\end{array}$ \\
$Q_{a}$ & $\frac{q_{a}^{N_{a}}}{N_{a} !} e^{\beta N_{a} E(\theta)}$ & $\frac{q_{a}^{N_{a}}}{N_{a} !} e^{\beta N_{a} E(\theta)}$ & $\frac{q_{a}^{N_{a}}}{N_{a} !\left(N_{s}-N_{a}\right) !} e^{\beta N_{a} E(\theta)}$ \\
$q_{a}$ & $\frac{A q_{v}}{\Lambda^{2}}$ & $\frac{\left(A-b N_{a}\right) q_{v}}{\Lambda^{2}}$ & $\frac{A q_{v}}{N_{s} \Lambda^{2}}$ \\
$P_{\mathrm{H}_{2}}^{e q}$ & $P_{i d}$ & $P_{i d}^{2 \theta(1-\theta)}$ & $P_{i d} \frac{1}{(1-\theta)^{2}}$ \\
\hline \hline
\end{tabular}

$$
\frac{N_{g}}{V}=\frac{q_{g}}{V}\left(\frac{e^{\rho \Lambda^{2}}-1}{q_{v}}\right)^{2} \frac{1}{\left(1-\theta / \theta_{M}\right)^{2}} e^{-2 \beta F(\theta)}
$$

and the equilibrium pressure

$$
\begin{aligned}
P_{\mathrm{H}_{2}}^{e q} & =k_{B} T \frac{q_{g}}{V}\left(\frac{e^{\rho \Lambda^{2}}-1}{q_{v}}\right)^{2} \frac{1}{\left(1-\theta / \theta_{M}\right)^{2}} e^{-2 \beta F(\theta)} \\
& =P_{i d} \frac{1}{\left(1-\theta / \theta_{M}\right)^{2}} .
\end{aligned}
$$

Predictions on Ir for selected values of pressure are reported in Figs. 2(g), 2(h), and 2(i).

In order to ease the comparison between the models considered, we summarize the main assumptions and conclusions for the case $\theta_{M}=1$ in Table V. From the results reported in Fig. 2 for the $\operatorname{Ir}(111)$ surface it is apparent that one must include some sort of saturation to obtain a correct qualitative description of surface coverage. This can be achieved by including it explicitly in the statistical treatment, as is the case for the hard disk and the anchored models, or through the fitting function, i.e., by considering binding energies that decrease sharply for high coverages (as in the tanh and hyperbolic fits). It is also apparent that the three models predict essentially the same results for coverages under $\approx 0.2$. We expect the behavior of the real system to be between the hard-disk model and the anchored model, depending on the specific system considered and the conditions used. Indeed these two models yield results that are essentially independent of the fitting function for temperatures above $400 \mathrm{~K}$.

\section{F. Adsorption and desorption}

In order to predict adsorption and desorption kinetics and the time evolution of $\theta$ it is necessary to have a detailed knowledge of the transition state for the process, but we do not have this information.

Even so, we can verify that our treatment of equilibrium is consistent with available experimental data by showing that plausible assumptions about the kinetics of the process lead to adsorption profiles and TPD spectra in good agree- 
ment with experiment. To this end, we derive a generic equation and investigate which assumptions must be satisfied to match the experimental observables.

We assume that the number of gas phase molecules hitting the surface per unit time is given by the equilibrium distribution of velocities, i.e., the presence of the surface does not perturb the thermal distribution in the gas phase. From transition state theory, the adsorption rate, in units of $N_{g} /($ s area $)$, is

$$
\begin{aligned}
\operatorname{rate}_{a} & =\mathcal{S} \frac{k_{B} T}{h}\left(\frac{2 \pi m_{\mathrm{H}_{2}} k_{B} T}{h^{2}}\right)^{-1 / 2}\left[\mathrm{H}_{2}\right] \\
& =\mathcal{S}\left(\frac{k_{B} T}{2 \pi m_{\mathrm{H}_{2}}}\right)^{1 / 2}\left[\mathrm{H}_{2}\right],
\end{aligned}
$$

where $\mathcal{S}$ is the sticking coefficient. The desorption rate must equal the adsorption rate at equilibrium coverage, hence we must have

$$
\text { rate }_{d}=\mathcal{S}\left(\frac{k_{B} T}{2 \pi m_{\mathrm{H}_{2}}}\right)^{1 / 2}\left[\mathrm{H}_{2}\right]_{e q},
$$

where $\left[\mathrm{H}_{2}\right]_{e q}$ is the equilibrium gas concentration corresponding to surface coverage $\theta$. In our treatment, $\left[\mathrm{H}_{2}\right]_{e q}$ $=P_{\mathrm{H}_{2}}^{e q} /\left(k_{B} T\right)$, where $P_{\mathrm{H}_{2}}^{e q}$ is given by Eqs. (19), (24), or (31). We stress the fact that $P_{\mathrm{H}_{2}}^{e q}$ is the theoretical gas phase pressure required to achieve a given level of surface coverage $\theta$ under equilibrium conditions. This corresponds to a measurable hydrogen partial pressure $P$ only in the special case that no net adsorption or desorption is under way, i.e., when the rates of adsorption and desorption are equal.

Since every adsorbed molecule results in two adsorbed atoms, the above rates must be doubled when the units of $N_{a} /$ (s area) are used. Considering that $\theta=\left[N_{a}\right] /\left[N_{s}\right]$, we obtain the time evolution of $\theta$ described by the differential equation

$$
\frac{d \theta}{d t}=\mathcal{S} \frac{2}{\left[N_{s}\right]}\left(\frac{k_{B} T}{2 \pi m_{\mathrm{H}_{2}}}\right)^{1 / 2}\left\{\left[\mathrm{H}_{2}\right]-\left[\mathrm{H}_{2}\right]_{e q}\right\} .
$$

The explicit form of Eq. (34) depends on the model used to describe $\left[\mathrm{H}_{2}\right]_{e q}$ and on the sticking coefficient $\mathcal{S}$.

Notice that $\mathcal{S}$ appears in both rate expressions. The desorption rate is expected to depend on $T$ and $\theta$ but not on $P$. Therefore, $\mathcal{S}$ cannot depend on $P$ or on other quantities which are functions of $P$, such as the equilibrium coverage. The sticking coefficient can thus depend only on $T, \theta$, and metal specific parameters, such as the maximum coverage $\theta_{M}$. Furthermore, $\mathcal{S}$ must be such that the rate of adsorption does not increase with coverage and the rate of desorption does not decrease with coverage. Hence, the permissible functional forms of $\mathcal{S}$ depend on the adopted surface model. We assume that the sticking coefficient $\mathcal{S}$ depends on the temperature via an Arrhenius-like expression,

$$
\mathcal{S}(T, \theta)=e^{E_{A} / R T} f(\theta),
$$

where $E_{A}$ is a metal dependent, temperature independent activation energy and $f(\theta)$ is a nonincreasing function of theta defined between 0 and $\theta_{M}$. We further assume that the stick- ing coefficient tends to unity in the limit of high temperature and low coverage. Therefore we impose the condition $f(0)$ $=1$. Based on the experimental values for the sticking coefficients, we expect $E_{A}$ to be at most a couple of $\mathrm{kcal} / \mathrm{mol}$.

\section{G. Rates using the ideal surface gas model}

In the case of an ideal surface gas, rate equation (34) becomes

$$
\begin{aligned}
\frac{d \theta}{d t}= & \mathcal{S} \frac{2}{\left[N_{s}\right]}\left(\frac{k_{B} T}{2 \pi m_{\mathrm{H}_{2}}}\right)^{1 / 2}\left\{\left[\mathrm{H}_{2}\right]-\left(\frac{q_{g}}{V}\right)\right. \\
& \left.\times\left(\frac{e^{\theta \Lambda^{2}\left[N_{s}\right]}-1}{q_{v}}\right)^{2} e^{-2 \beta F(\theta)}\right\} .
\end{aligned}
$$

Since the ideal surface gas has no volume, the only plausible choice for the sticking coefficient consistent with our assumptions is $\mathcal{S}(T, \theta)=\mathcal{S}(T)=e^{E_{A} / R T}$. In particular $\mathcal{S}$ is constant at constant temperature. The predicted adsorption profile for $\operatorname{Ir}(111)$ at $100 \mathrm{~K}$ with linear, tanh, and hyperbolic fit is reported in Figs. 3(a), 3(b), and 3(c), where two values of $\mathcal{S}$ are reported for the linear fit case. In each case we compare a fit to the experimental adsorption profile. ${ }^{33}$ It is apparent that the agreement between prediction and experiment is extremely poor for all ideal gas models, which are expected to be accurate only for values of $\theta$ tending to zero. In this regime the comparison between theory and experiment leads to an estimate for the sticking coefficient of $\approx 0.015$. This value corresponds to an activation energy $E_{A}$ $=0.83 \mathrm{kcal} / \mathrm{mol}$.

\section{H. Rates using the hard-disk model}

In the case of hard-disk model, the kinetic equation is

$$
\begin{aligned}
\frac{d \theta}{d t}= & \mathcal{S} \frac{2}{\left[N_{s}\right]}\left(\frac{k_{B} T}{2 \pi m_{\mathrm{H}_{2}}}\right)^{1 / 2}\left\{\left[\mathrm{H}_{2}\right]-\left(\frac{q_{g}}{V}\right)\right. \\
& \times\left(\frac{e^{\rho \Lambda^{2}}-1}{q_{v}}\right)^{2}\left(\frac{e^{\theta /\left(\theta_{M}-\theta\right)}}{1-\theta / \theta_{M}}\right)^{2} e^{-2 \beta F(\theta)} .
\end{aligned}
$$

Since the surface atoms have a well defined area in this model, we expect the sticking coefficient for dissociative chemisorption to be proportional to the square of the available area, i.e, $\mathcal{S}(T, \theta)=e^{E_{A} / R T}\left(1-\theta / \theta_{M}\right)^{2}$. This functional form is acceptable since the desorption rate increases with $\theta$ and, in particular, it does not vanish for $\theta=\theta_{M}$. Thus we use the expression

$$
\begin{aligned}
\frac{d \theta}{d t}= & \frac{2 e^{E_{A} / R T}}{\left[N_{s}\right]}\left(\frac{k_{B} T}{2 \pi m_{\mathrm{H}_{2}}}\right)^{1 / 2}\left\{\left(1-\theta / \theta_{M}\right)^{2}\left[\mathrm{H}_{2}\right]-\left(\frac{q_{g}}{V}\right)\right. \\
& \left.\times\left(\frac{e^{\rho \Lambda^{2}}-1}{q_{v}}\right)^{2} e^{2 \theta /\left(\theta_{M}-\theta\right)} e^{-2 \beta F(\theta)}\right\} .
\end{aligned}
$$

The corresponding adsorption profile and TPD spectra are reported in Figs. 3(d), 3(e), and 3(f) and 4(d), 4(e), and 4(f). 
(A) Ideal gas - Linear fit

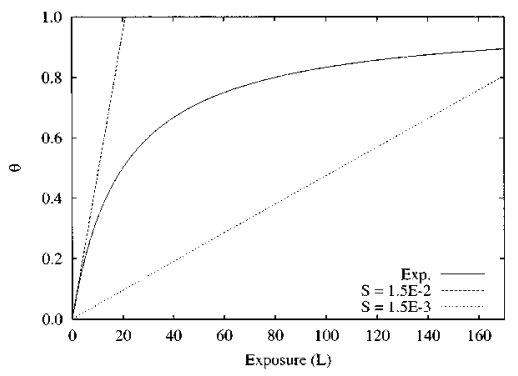

(B) Ideal gas - Tanh fit

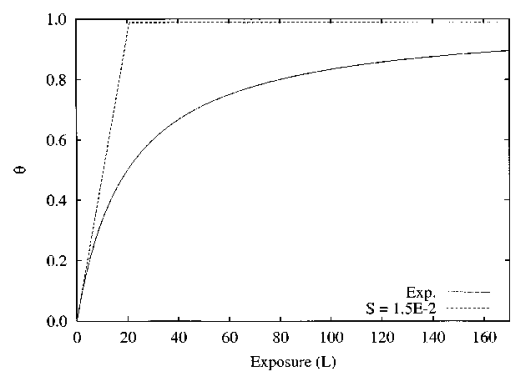

(C) Ideal gas - Hyperbolic fit

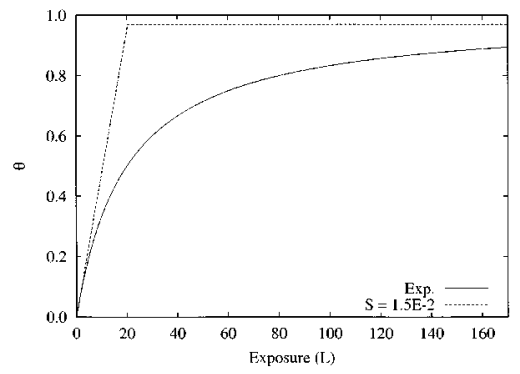

(D) Hard Disk gas - Linear fit

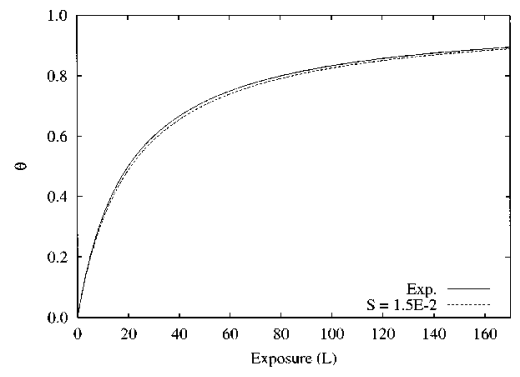

(E) Hard Disk gas - Tanh fit

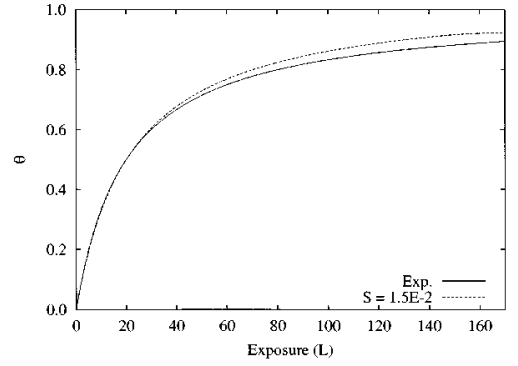

(F) Hard Disk gas - Hyperbolic fit

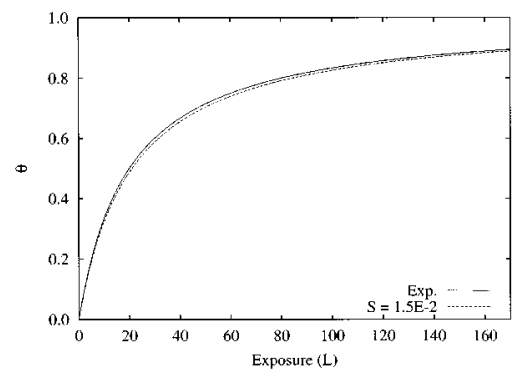

(G) Anchored - Linear fit

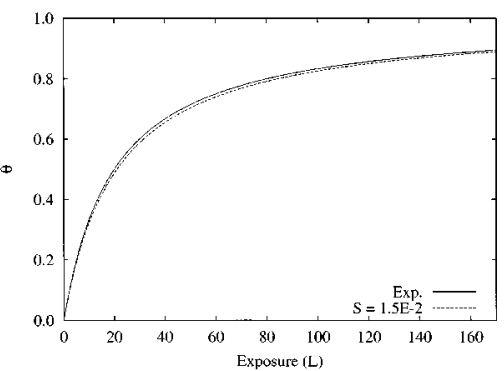

(H) Anchored - Tanh fit

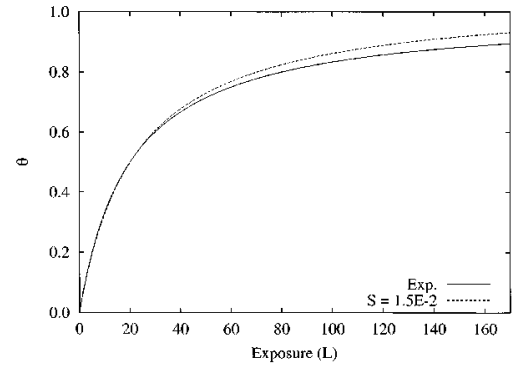

(I) Anchored - Hyperbolic fit

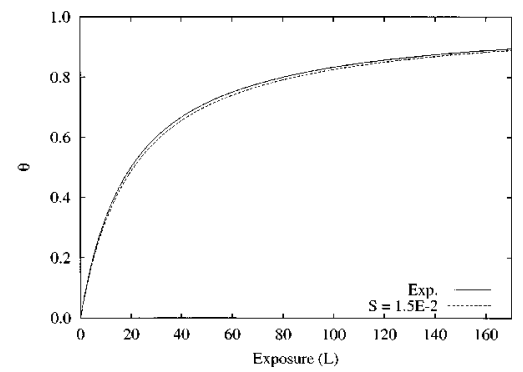

FIG. 3. Adsorption profiles on $\operatorname{Ir}(111)$ at $100 \mathrm{~K}$ computed with various statistical models and fitting functions. The solid line is a fit to experimental (Ref. 33). Exposures are in Langmuir $\left(1 \mathrm{~L}=1.31579 \times 10^{-9} \mathrm{~atm} \mathrm{~s}\right)$. The ideal gas fails to produce the correct behavior because it does not include saturation explicitly which imposes strong constraints on the form of the sticking coefficient. The best agreement with experiment is given by the hard-disk or anchored model using the hyperbolic or linear fits. 


\section{Rates using the anchored model}

For the anchored hydrogen model, we can use the same sticking coefficient as for the hard-disk model, resulting in the following kinetic equation:

$$
\begin{aligned}
\frac{d \theta}{d t}= & \frac{2 e^{E_{A} / R T}}{\left[N_{s}\right]}\left(\frac{k_{B} T}{2 \pi m_{\mathrm{H}_{2}}}\right)^{1 / 2}\left\{\left(1-\theta / \theta_{M}\right)^{2}\left[\mathrm{H}_{2}\right]\right. \\
& \left.-\frac{q_{g}}{V}\left(\frac{e^{\rho \Lambda^{2}}-1}{q_{v}}\right)^{2} e^{-2 \beta F(\theta)}\right\} .
\end{aligned}
$$

The corresponding adsorption profiles and TPD spectra are reported in Figs. 3(g), 3(h), and 3(i) and 4(g), 4(h), and 4(i).

\section{J. Discussion of predicted rates}

Figure 3 shows that the ideal gas model cannot reproduce the experimental behavior even qualitatively. In this model the only possible form of the sticking coefficient is a constant (at constant temperature) so that the desorption rate does not decrease with coverage. In addition, as noted above for the equilibrium results, the ideal gas model fails for coverages greater than $\approx 0.2 \mathrm{ML}$.

The hard-disk and anchored models both allow more reasonable forms of the sticking coefficient and they both reproduce well the experimental curve.

Figure 4 shows that all predicted TPD spectra exhibit a shift of the peaks towards lower temperature for increasing surface coverages. This shift is quite modest for the tanh fit and much larger for both the linear and hyperbolic fits. The experimental shift between 1 and $20 \mathrm{~L}$ is approximately of $60 \mathrm{~K} \cdot{ }^{34}$ For the same two exposures we compute shifts using the tanh fit of $33 \mathrm{~K}$ (anchored and ideal gas models) and 43 $\mathrm{K}$ (hard-disk model). The linear and hyperbolic fits are indistinguishable in this region and they yield shifts of $78 \mathrm{~K}$ (anchored and ideal gas models) and $91 \mathrm{~K}$ (hard-disk model). We predict high coverage, low temperature shoulders in the TPD spectra for all three models using the tanh fit and for all three coverage dependencies for the hard-disk gas model. We expect the shape of this shoulder to depend on the form of the fitting function used for $E(\theta)$. The TPD spectra reported in Fig. 4 refer to initial surface coverages $\theta$ between 0.02 and the saturation value for each model at a pressure of $1.31579 \times 10^{-9} \mathrm{~atm}$. A less marked but still evident shoulder is predicted in the case of hard-disk surface gas model even when a linear fit to the binding energy is used. This indicates that the shoulder could arise from the behavior of the adsorbed hydrogen even when no weakly bound species are present.

These results show that the overall shape of the TPD spectrum is affected substantially by the surface model and fitting function used. In the case of Ir, where we expect hydrogen to diffuse extremely easily on the surface, the model that appears to best reproduce the experimental spectra is the hard-disk model with either the linear or hyperbolic fit to the binding energy. However, it is possible that different models or fitting functions might be more appropriate for other metals.

\section{K. Surface coverage for other metals}

We report in this section the predicted equilibrium surface coverages for all nine metals considered based on the hard-disk statistical model and the linear fit to the energy. Although this may not be the most appropriate combination to describe surface science experiments, in the case of high temperature catalytic processes hydrogen is expected to diffuse relatively fast on the metal surface, suggesting the harddisk model as the most appropriate tool among those developed in this document. Furthermore, under catalytic conditions, it is customary to assume there are available sites on the surface for other species to adsorb and react. It is thus of limited interest to investigate coverages near one monolayer. Also, for intermediate coverages the adsorbed hydrogen in expected to diffuse between different adsorption sites without necessarily occupying the most stable and ordered configuration. This suggests that the linear fit to the binding energy may provide an appropriate description.

We report the estimated coverages for the surfaces stable at room temperature in Fig. 5. The unstable $\mathrm{Fe}$ and Co surfaces are reported in Fig. 6.

\section{TPD interpretation}

It is instructive to analyze the TPD spectra obtained from our simulations with the common tools used to interpret experimental TPD spectra, i.e., the generalizations of Redhead method $^{35}$ based on Polanyi-Wigner equation. Since we know the exact form of $E(\theta)$ used to produce the spectra, the values extracted from this analysis will provide an estimate of the error associated with the interpretation of experimental data.

The basic equation of the heating rate variation method for second-order desorption is

$$
\ln \frac{T_{M}^{2}}{\beta}=\frac{E^{\ddagger}}{R T_{M}}+\ln \frac{E^{\ddagger}}{\nu R \theta_{0}},
$$

where $T_{M}$ is the peak temperature, $\beta$ is the heating rate, $\theta_{0}$ is the initial surface coverage, $E^{*}$ is the activation energy, and $\nu$ is the preexponential factor for the Arrhenius-like kinetic equation describing the desorption process.

Consider, for instance, the behavior of Ir with the harddisk model and the linear fit, with simulated TPD spectrum reported in Fig. 4(d). In this case, the enthalpy of adsorption for a gas phase $\mathrm{H}_{2}$ molecule at $0 \mathrm{~K}$ is given by the linear fit (in $\mathrm{kcal} / \mathrm{mol}$ )

$$
E(\theta)=17.1-3.0 \theta \text {. }
$$

This was obtained from the fit reported in Table IV. Since it refers to two surface atoms forming one gas phase molecule, the expression from Table IV was doubled and the bond energy of $\mathrm{H}_{2}$ at $0 \mathrm{~K}$, or $103.24 \mathrm{kcal} / \mathrm{mol}$, was subtracted. Also, in the simulation we used an activation energy for the sticking coefficient of $0.8 \mathrm{kcal} / \mathrm{mol}$.

We simulated the TPD spectra with heating rates of 1,2, $5,10,20$, and $50 \mathrm{~K} / \mathrm{s}$. For each value of initial surface coverage, we obtained the linear least squares fit to $\ln T_{M}^{2} / \beta$ versus $1 / T_{M}$. According to Eq. (40), one can extract the values for $E^{\ddagger}$ and $\nu$ from the slope and the intercept. The activation energies obtained for various initial coverages are reported in 
(A) Ideal gas - Linear fit

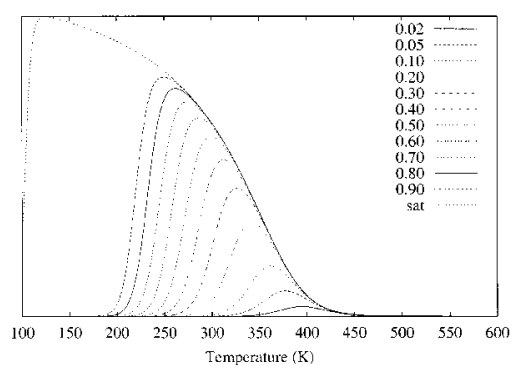

(B) Ideal gas - Tanh fit

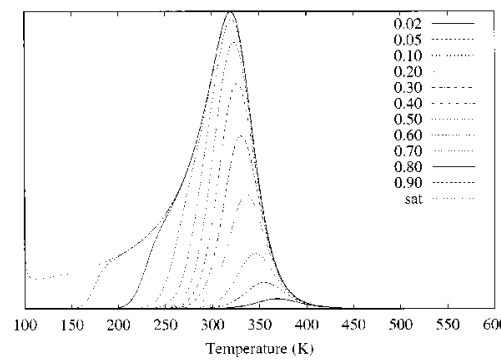

(C) Ideal gas - Hyperbolic fit

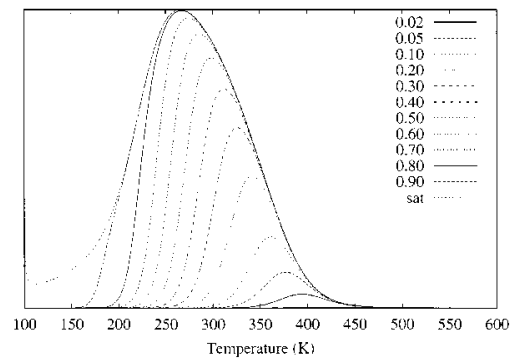

(D) Hard Disk gas - Linear fit

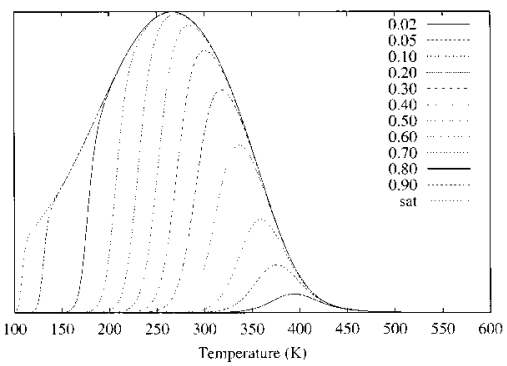

(E) Hard Disk gas - Tanh fit

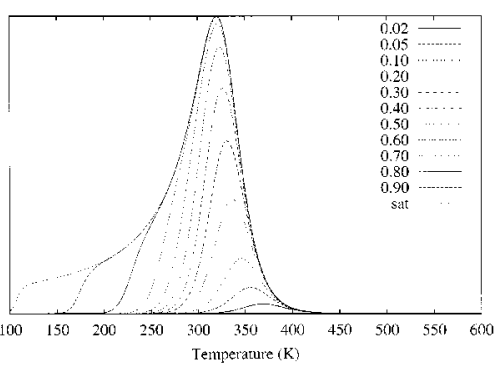

(F) Hard Disk gas - Hyperbolic fit

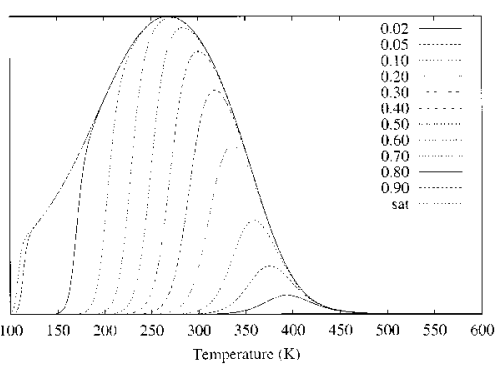

(G) Anchored - Linear fit

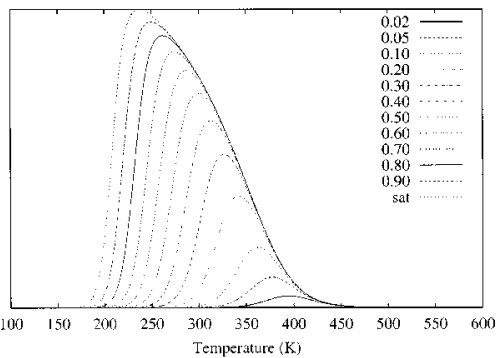

(H) Anchored - Tanh fit

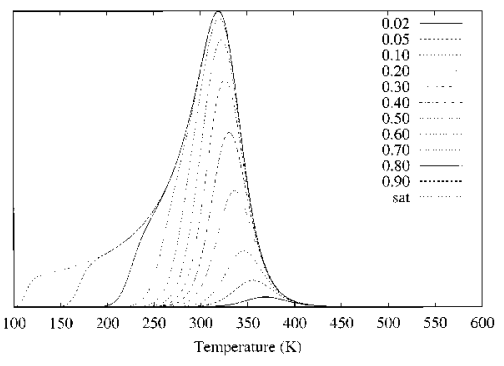

(I) Anchored - Hyperbolic fit

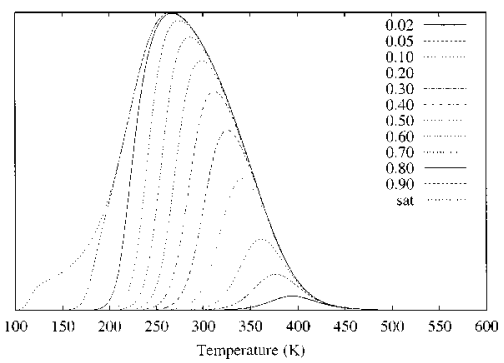

FIG. 4. TPD spectra on $\operatorname{Ir}(111)$ computed with various models and fitting functions. Starting coverages are in units of $\theta$ (saturation values refer to a loading pressure of $\left.1.31579 \times 10^{-9} \mathrm{~atm}\right)$. Heating rate is $20 \mathrm{~K} / \mathrm{s}$. The shape of the spectra depends on both the fitting function and the model. Thus comparison of such results with experiment can provide a criterion for selecting the model and fitting function. All nine cases lead to a single peak that shifts with increasing coverage, but the shift is smaller for the tanh fit. The hard-disk model leads to a low temperature shoulder at high coverage for all fitting functions. Probably the hard-disk model with a hyperbolic fit best compares with experiment. 
$\mathrm{Fe}$

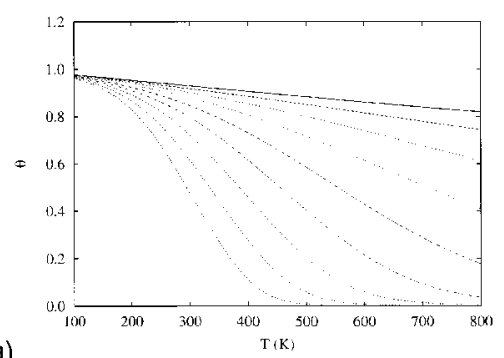

(a)

Co

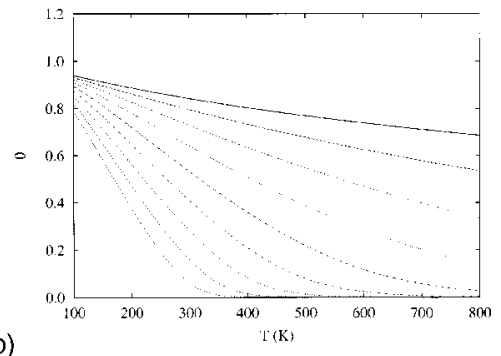

(b)

(c)

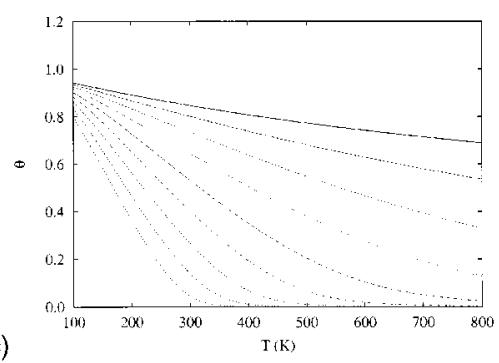

Ru

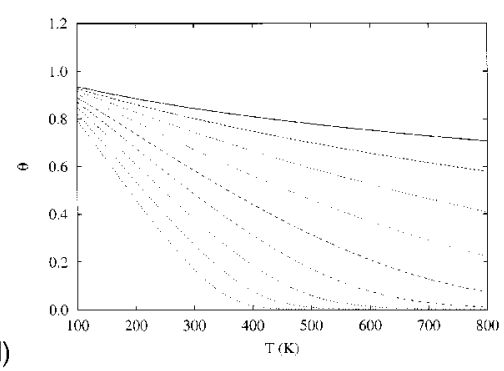

$\mathrm{Rh}$

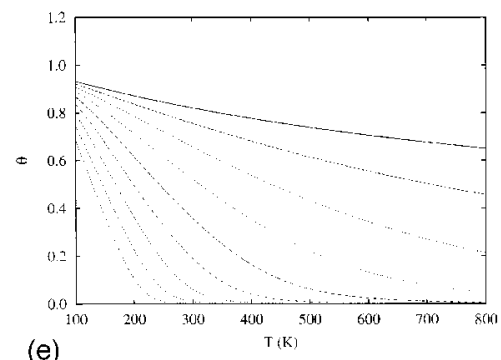

$\mathrm{Pd}$

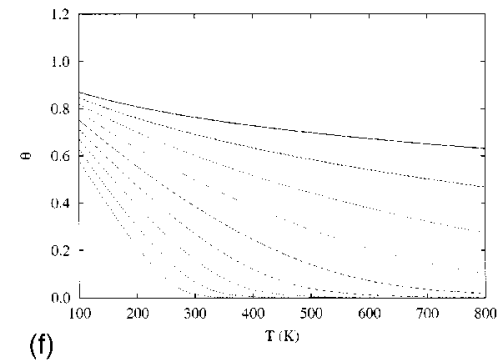

Os

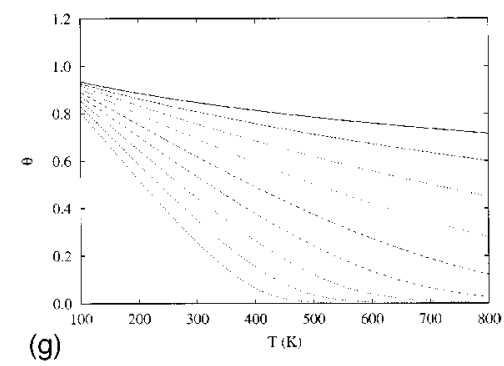

Ir

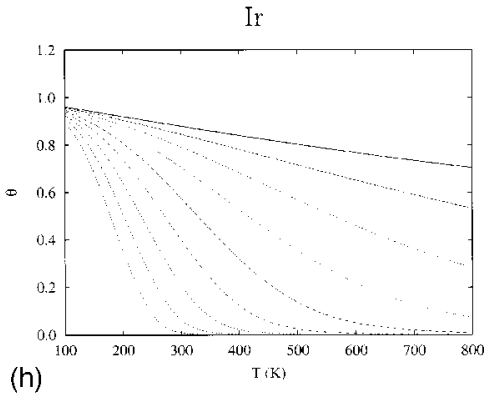

(h)

$\mathrm{Pt}$

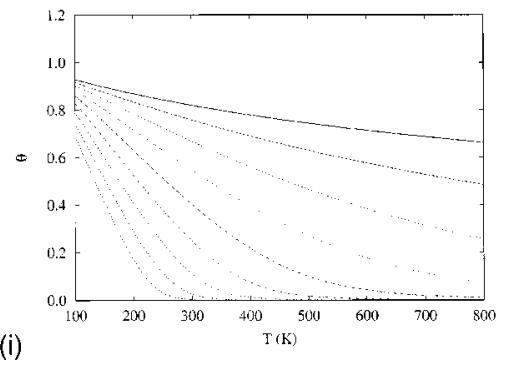

FIG. 5. Predicted equilibrium surface coverage $\theta$ for the nine stable surfaces at ambient conditions as a function of temperature using the linear fit with the hard-disk model. Each line corresponds to one of nine selected values of pressure: $10^{-14}$ (lower, leftmost line), $10^{-12}, 10^{-10}, 10^{-8}, 10^{-6}, 10^{-4}, 10^{-2}, 10^{0}$, and $10^{2} \mathrm{~atm}$ (upper, rightmost line).

Table VI, where we also provide the differences between the activation energy $E^{\ddagger}$ and both the exact binding energy $E(\theta)$ and the corresponding $F(\theta)=E(\theta)+\theta d E / d \theta$. Thus we would expect $E^{\ddagger}$ at concentration $\theta$ to be related to $F(\theta)$. Indeed, for $\theta$ between 0.02 and 0.5 they differ by $\approx 1 \mathrm{kcal} /$ mol, which is close to the chemisorption activation energy of $0.8 \mathrm{kcal} / \mathrm{mol}$ which we assumed for the simulation in Fig. 4(d).

For $\theta$ greater than 0.5 the deviation in $E^{\ddagger}-F(\theta)$ increases apparently because of deviation from the Polanji- 


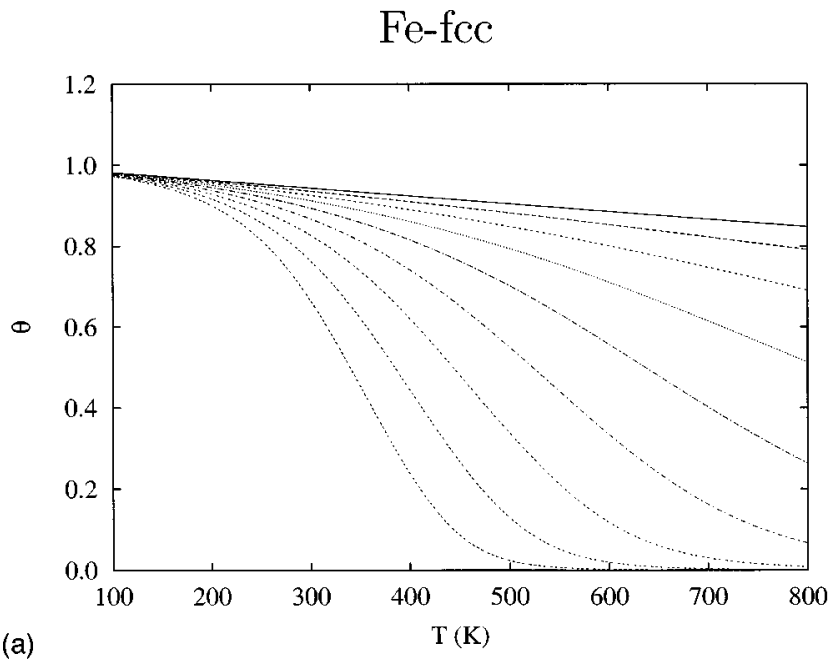

(a)

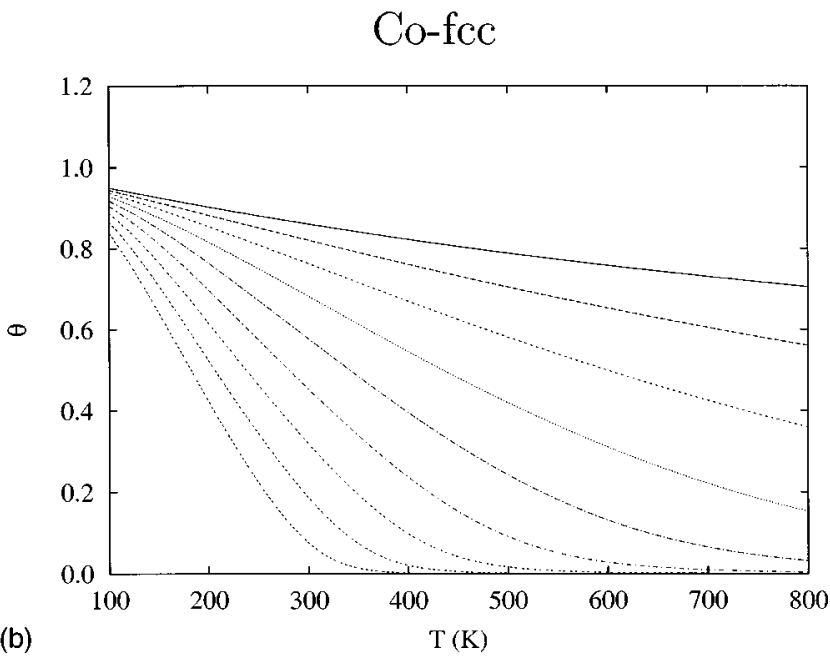

FIG. 6. Predicted equilibrium surface coverage $\theta$ as a function of temperature using the linear fit with the hard-disk model. Each line corresponds to one of nine selected values of pressure: $10^{-14}$ (lower, leftmost line), $10^{-12}$, $10^{-10}, 10^{-8}, 10^{-6}, 10^{-4}, 10^{-2}, 10^{0}$, and $10^{2}$ atm (upper, rightmost line).

Wigner expression assumed in the Redhead analysis.

We conclude that, at least for this model of TPD which is based on equilibrium statistical thermodynamics, the commonly used interpretation methods based on the Polanji-

TABLE VI. Activation energies $E^{\ddagger}$ and preexponential factors $\nu$ obtained from the interpretation of the TPD spectra in Fig. 4(d) with a Redhead-like approach, for various initial surface coverages $\theta_{0}$. The last two columns contain the difference with respect to $E(\theta)$, from Eq. (41), and $F(\theta)$ $=E(\theta)+\theta(d E / d \theta)$.

\begin{tabular}{ccccc}
\hline \hline $\begin{array}{c}\theta_{0} \\
(\mathrm{ML})\end{array}$ & $\begin{array}{c}E^{\ddagger} \\
(\mathrm{kcal} / \mathrm{mol})\end{array}$ & $\begin{array}{c}\nu \\
\left(\mathrm{ML}^{-1} \mathrm{~s}^{-1}\right)\end{array}$ & $\begin{array}{c}E^{\ddagger}-E(\theta) \\
(\mathrm{kcal} / \mathrm{mol})\end{array}$ & $\begin{array}{c}E^{\ddagger}-F(\theta) \\
(\mathrm{kcal} / \mathrm{mol})\end{array}$ \\
\hline 0.02 & 17.9 & $4.8 \times 10^{9}$ & 0.9 & 0.9 \\
0.05 & 17.8 & $5.4 \times 10^{9}$ & 0.8 & 1.0 \\
0.10 & 17.5 & $6.0 \times 10^{9}$ & 0.7 & 1.0 \\
0.20 & 16.9 & $7.3 \times 10^{9}$ & 0.4 & 1.0 \\
0.30 & 16.4 & $9.8 \times 10^{9}$ & 0.2 & 1.1 \\
0.40 & 15.8 & $1.4 \times 10^{10}$ & -0.1 & 1.1 \\
0.50 & 15.3 & $2.1 \times 10^{10}$ & -0.3 & 1.2 \\
0.60 & 15.1 & $5.4 \times 10^{10}$ & -0.2 & 1.6 \\
0.70 & 15.5 & $1.6 \times 10^{11}$ & 0.5 & 2.6 \\
\hline \hline
\end{tabular}

Wigner equation, which, in turn, is based on the Arrhenius form for the kinetics of the desorption process, provide an estimate to the function $F(\theta)$ rather than $E(\theta)$. The estimate thus obtained, however, includes the activation energy for the adsorption process. Consequently, it provides only an upper bound to the enthalpy of chemisorption. In order to extract the $\mathrm{BE}$ from this data, it is thus necessary to estimate the adsorption barrier.

\section{Comparison with experiment}

A general overview of experimental results for hydrogen adsorption on group VIII transition metals is given in several reviews. ${ }^{3-5}$ We discuss here only published results relevant for discussing and interpreting our computations.

TPD spectra and adsorption profiles for hydrogen were obtained on $\mathrm{Fe},{ }^{36,37} \mathrm{Ni},{ }^{38-41} \mathrm{Ru},{ }^{42-44} \mathrm{Rh},{ }^{45} \mathrm{Pd},{ }^{46,47} \mathrm{Ir},{ }^{19,34,48}$ and Pt. ${ }^{49,50}$ All TPD spectra appear to have one peak at low coverages. The peak shifts towards lower temperatures with increasing initial exposure. This agrees with essentially all models in Fig. 4.

A second peak or shoulder appears in the low temperature region of the spectra for high initial coverages [e.g., above $150 \mathrm{~L}$ for $\mathrm{Ni}$ (Ref. 39) and above 100-160 L for Ir (Refs. 19, 34, and 48)]. Our results for the hard-disk model (all three fits) and for the tanh fit (all three surface models) lead to a pronounced shoulder in agreement with many of these results. However, we do not find the low temperature peak observed for some surfaces, namely, Fe and Ni. This peak is associated with a phase transition in the adsorbed hydrogen. ${ }^{3,39,51}$ On other surfaces, it is associated with metal hydrides near the surface. ${ }^{47}$ While the low temperature, high coverage phase is not likely to play any significant role in catalytic processes, it may affect significantly the surface science experiments, raising doubts on the applicability of surface science conclusions to working catalysts. In some systems, the shape of the second peak or shoulder depends strongly on the conditions used in cleaning the surface and in performing the measurement. ${ }^{52}$

There is general agreement that the surface binding energy depends on coverage, but there is no universally accepted model to fully interpret the TPD results. Important quantities such as binding energies and desorption barriers depend somewhat on the assumptions made in order to interpret the spectra. We suggest that the hyperbolic form might best describe both the theory and experiment.

The binding site of adsorbed hydrogen has been probed using low-energy electron diffraction (LEED), electronenergy-loss spectroscopy (EELS), and other similar techniques. Except for $\operatorname{Ir}(111)$ hydrogen appears to prefer threefold cap positions on all closest packed surfaces. For Ir, there is evidence of adsorption in the top position. ${ }^{19}$ Indeed this is consistent with our calculations which lead to a top site for Ir (by $1.4 \mathrm{kcal} / \mathrm{mol}$ ) and to fcc sites for all other metals. Indeed for the $3 d$ and $4 d$ transition metals, the calculated binding to the top sites is too weak to dissociate $\mathrm{H}_{2}$, while for all three $5 d$ cases the top site is competitive with fcc (for Pt and Os it is 0.2 and $2.1 \mathrm{kcal} / \mathrm{mol}$ higher).

On some metals (e.g., Ir and Rh) at low surface coverage hydrogen is considered to be delocalized. ${ }^{19,53}$ 
In the low coverage region of the $\operatorname{Ir}(111)$ TPD spectra our results agrees well with experiments. ${ }^{34}$ For example, an exposure of $1 \mathrm{~L}$ yields an experimental peak around $350 \mathrm{~K}$ and corresponds roughly to $\theta=0.1$, which in our TPD simulation peaks around $350 \mathrm{~K}$. Also, with the linear and hyperbolic fits we predict the spectral peak close to the experimentally observed value of $250 \mathrm{~K}$. We find that a low temperature shoulder in the spectra can result from saturation effects in the model or from a decreasing binding energy at high coverages. We stress the fact that the purpose of this paper is not to reliably predict TPD spectra. The agreement obtained between prediction and experiment was achieved by arbitrarily selecting the shape of the binding energy fitting function and the adsorption model. This only shows that the computed energies are consistent with the observed TPD spectra.

For other metals, other assumptions may be appropriately selected to reproduce the observed TPD spectra. For instance, the models considered so far are unable to predict a second peak in the spectra, as observed, depending on experimental conditions and sample history for $\mathrm{Fe}$ and Ni. A suitable modification of the fitting function may result in the appearance of two peaks in the TPD spectra.

In the case of $\mathrm{Ni}$, for instance, the experimental spectra have one high temperature peak around $400 \mathrm{~K}$ and a low temperature peak assigned around 300 (Ref. 40 ) or $350 \mathrm{~K}^{41}$ We can reproduce these peaks assuming an activation energy for the adsorption sticking coefficient of $3.0 \mathrm{kcal} / \mathrm{mol}$ and using the following functional form for the binding energy:

$$
E(\theta)=60.5-0.6 \theta-0.1 \tanh \left[10\left(\theta-\frac{1}{2}\right)\right],
$$

which passes within the error bar of the computed values up to ML coverage. To obtain this curve, we adjusted the $\mathrm{BE}$ by increasing it by $0.1,1.1$, and $1.5 \mathrm{kcal} / \mathrm{mol}$ for $1 / 3,2 / 3$, and $3 / 3$ ML coverages, respectively. We report the corresponding TPD spectrum in Fig. 7 for the hard-disk model.

Similar procedures can be applied to reproduce the main features of all other experimental spectra. Our models do not allow for phase changes in the adsorbate, which are widely believed to be responsible for the presence of multiple peaks in TPD spectra of closest packed surfaces. Also, our models are not designed to describe multilayer and subsurface adsorption, which may also affect TPD spectra by adding low temperature peaks and by changing the shape of the high temperature portion of the spectra depending on exposure and history of the sample. To describe such effects it is necessary to use more sophisticated treatments tuned to the metal of interest. However, consistency of the computed binding energies interpreted with the hard-disk and anchored models with the observed adsorption and TPD profiles, suggests that plausible assumptions can reconcile the observed spectra with our computed binding energies. We conclude as follows:

(A) The observation of low temperature shoulders does not prove the presence of two species or two phases on the surface or a stepped binding energy dependence on coverage. Of course, these effects would also result in shoulders and new peaks in the spectra. This indicates that the presence of

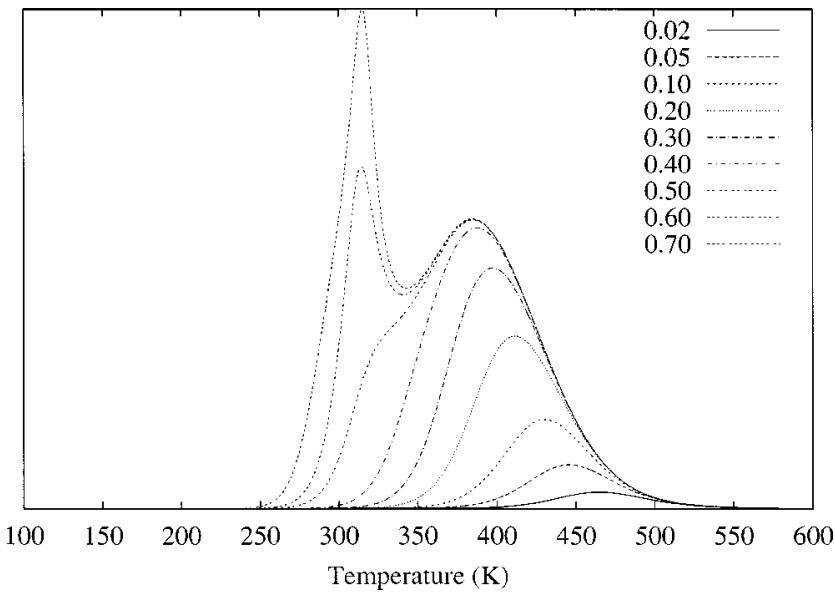

FIG. 7. Simulated TPD spectra for $\mathrm{H}_{2}$ desorption from Ni(111). The harddisk thermodynamics model is applied with the energy profile 42 . Heating rate is $10 \mathrm{~K} / \mathrm{s}$. Each line corresponds to a different initial surface coverage as indicated in the legend.

a shoulder cannot be taken as conclusive evidence to establish the nature of adsorbed hydrogen.

(B) The computed binding energies are not in contrast with the reported TPD spectra.

All predicted equilibrium surface coverages in Fig. 2, except for the ideal gas model with linear fit, are qualitatively acceptable, but the corresponding adsorption profiles and TPD spectra differ significantly. In particular, to describe correctly the adsorption profile some form of explicit saturation effect must be included. In fact the ideal gas model provides a wrong adsorption profile even when strong interatomic interactions are included for high coverages via the binding energy dependence on $\theta$.

\section{CONCLUSIONS}

We use density functional theory to investigate hydrogen coverage of the most stable surfaces of group VIII transition metals. In particular, we studied how the binding energy depends on the specific metal and surface coverage. These DFT results agree well with experimental measures with average errors of $2.0 \mathrm{kcal} / \mathrm{mol}$ in the heat of formation of adsorbed hydrogen and $6 \%$ in the vibrational frequencies. Thus the calculations match published data within experimental accuracy.

We report a simple statistical treatment of the adsorbate to validate the consistency of our results with available adsorption profiles and TPD spectra. Explicit estimates of hydrogen surface coverage at temperatures and pressures typical of catalytic conditions provide two limiting situations: high temperature with a low diffusion barrier and low temperature with a high diffusion barrier. These estimates are expected to bracket the real surface coverage.

\section{ACKNOWLEDGMENTS}

The authors acknowledge NCSA for computational resources. During the period of this research the facilities of the MSC were supported by grants from DOE-ASCI, ARO/ DURIP, ARO/MURI, BP Chemical, Beckman Institute, 
Seiko-Epson, Exxon-Mobil, Owens-Corning, AveryDennison, Asahi Chemical, Chevron Petroleum Technology Co., Chevron Chemical Co., and Chevron Research and Technology Corp. Financial support was also provided by the European Research and Training Network "Molecular Properties and Materials (MOLPROP)," Contract No. HPRN-CT-2000-00013, and from the Italian MURST (Ministero dell'Università e della Ricerca Scientifica), via $60 \%$ and $40 \%$ funds. Supplementary material contains the software used to perform the equilibrium and kinetic simulations and the data files used to construct the figures.

${ }^{1}$ N. C. Satterfield, Heterogeneous Catalysis in Industrial Practice (McGraw-Hill, New York, 1991).

${ }^{2}$ B. C. Gates, Catalytic Chemistry (Wiley, New York, 1992).

${ }^{3}$ K. Christmann, Surf. Sci. Rep. 9, 1 (1988).

${ }^{4}$ J. W. Davenport and P. J. Estrup, in Chemisorption Systems: Part A and B, edited by D. A. King and D. P. Woodruff (Elsevier, Amsterdam, 1990), Vol. 3, Chap. 1.

${ }^{5}$ G. A. Somorjai, Introduction to Surface Chemistry and Catalysis (WileyInterscience, New York, 1994).

${ }^{6}$ The density functional GGA is commonly known as PW91. It contains Slater local exchange (Ref. 54), Perdew-Wang '91 correlation and nonlocal exchange (Refs. 55 and 56).

${ }^{7}$ CASTEP, versions 3.8 and 4.2.1, as described in Ref. 57.

${ }^{8}$ CERIUS2, version 4.0, Molecular Simulations, Inc.

${ }^{9}$ J. S. Lin, A. Qteish, M. C. Payne, and V. Heine, Phys. Rev. B 43, 4174 (1993).

${ }^{10}$ N. Troullier and J. L. Martins, Phys. Rev. B 43, 1993 (1991).

${ }^{11}$ N. Troullier and J. L. Martins, Phys. Rev. B 43, 8861 (1991).

${ }^{12}$ J. F. Paul and P. Sautet, Phys. Rev. B 53, 8015 (1996).

${ }^{13}$ A. Kokalj, A. Lesar, M. Hodošcek, and M. Causà, J. Phys. Chem. B 103, 7222 (1999).

${ }^{14}$ D. Vanderbilt, Phys. Rev. B 41, 7892 (1990).

${ }^{15}$ L. Hammer, H. Landskron, W. Nichtl-Pecher, A. Fricke, K. Heinz, and K. Müller, Phys. Rev. B 47, 15969 (1993).

${ }^{16}$ W. Nichtl-Pecher, G. Gossman, L. Hammer, K. Heinz, and K. Müller, J. Vac. Sci. Technol. A 10, 501 (1992).

${ }^{17}$ A. D. Johnson, K. J. Maynard, S. P. Daley, Q. Y. Yang, and S. T. Ceyer, Phys. Rev. Lett. 67, 927 (1991).

${ }^{18}$ H. Yanagita, H. Fujioka, T. Aruga, N. Takagi, and M. Nishijima, Surf. Sci. 441, 507 (1999).

${ }^{19}$ C. J. Hagedorn, M. J. Weiss, and W. H. Weinberg, Phys. Rev. B 60, R14016 (1999).

${ }^{20}$ P. Nordlander, S. Holloway, and J. K. Nørskov, Surf. Sci. 136, 59 (1984).

${ }^{21}$ D. E. Jiang and E. A. Carter, Surf. Sci. 547, 85 (2003).

${ }^{22}$ J. Greeley and M. Mavrikakis, Surf. Sci. 540, 215 (2003).

${ }^{23}$ G. Kresse and J. Hafner, Surf. Sci. 459, 287 (2000).

${ }^{24}$ I. M. Ciobică, R. A. Santen, A. W. Kleyn, and J. Hafner, Chem. Phys. Lett. 311, 185 (1999).
${ }^{25}$ P. Liu, A. Logadottir, and J. K. Nørskov, Electrochim. Acta 48, 3731 (2003).

${ }^{26}$ M. Mavrikakis, J. Rempel, J. Greeley, L. B. Hansen, and J. K. Nørskov, J. Chem. Phys. 117, 6737 (2002).

${ }^{27}$ D. X. W. Lai, Surf. Sci. 550, 15 (2004).

${ }^{28}$ W. Dong and J. Hafner, Phys. Rev. B 56, 15396 (1997).

${ }^{29}$ V. Pallassana, M. Neurock, L. B. Hansen, B. Hammer, and J. Nørskov, Phys. Rev. B 60, 6146 (1999).

${ }^{30}$ W. P. Krekelberg, J. Greeley, and M. Mavrikakis, J. Phys. Chem. B 108, 987 (2004).

${ }^{31}$ P. Légaré, Surf. Sci. 559, 169 (2004).

${ }^{32}$ J. R. Engstrom and W. H. Weinberg, J. Chem. Phys. 87, 4211 (1987).

${ }^{33}$ The data from Ref. 34 were fit to a second-order Langmuir model obtaining an initial probability of adsorption of 0.05 instead of the reported value of 0.0071 .

${ }^{34}$ J. R. Engstrom, W. Tsai, and W. H. Weinberg, J. Chem. Phys. 87, 3104 (1987).

${ }^{35}$ P. A. Redhead, Vacuum 12, 203 (1962).

${ }^{36}$ F. Bozso, G. Ertl, M. Grunze, and M. Weiss, Appl. Surf. Sci. 1, 103 (1977).

${ }^{37}$ J. Benziger and R. J. Madix, Surf. Sci. 94, 119 (1980).

${ }^{38}$ K. Christmann, O. Schober, and M. Neumann, J. Chem. Phys. 60, 4528 (1974).

${ }^{39}$ K. Christmann, R. J. Behm, G. Ertl, M. A. V. Hove, and W. H. Weinberg, J. Chem. Phys. 70, 4168 (1979).

${ }^{40}$ R. J. Behm, G. Ertl, M. A. V. Hove, and W. H. Weinberg, J. Chem. Phys. 70, 4168 (1979).

${ }^{41}$ A. Winkler and K. D. Rendulic, Surf. Sci. 118, 19 (1982).

${ }^{42}$ L. R. Danielson, M. J. Dresser, E. E. Donaldson, and J. T. Dickinson, Surf. Sci. 71, 599 (1978).

${ }^{43}$ H. Shimizu, K. Christmann, and G. Ertl, J. Catal. 61, 412 (1980).

${ }^{44}$ R. Feulner and D. Menzel, Surf. Sci. 154, 465 (1985).

${ }^{45}$ J. T. Yates, Jr., P. A. Thiel, and W. H. Weinberg, Surf. Sci. 84, 427 (1979).

${ }^{46}$ H. Conrad, G. Ertl, and E. E. Latta, Surf. Sci. 41, 435 (1974).

${ }^{47}$ G. E. Gdownski, T. E. Felter, and R. H. Stulen, Surf. Sci. 181, L147 (1987).

${ }^{48}$ P. D. Szuromi, J. R. Engstrom, and W. H. Weinberg, J. Chem. Phys. 80, 508 (1984).

${ }^{49}$ K. Christmann, G. Ertl, and T. Pignet, Surf. Sci. 54, 365 (1976).

${ }^{50}$ R. W. McCabe and L. D. Schmidt, Surf. Sci. 65, 189 (1977).

${ }^{51}$ R. Imbihl, R. J. Behm, K. Christmann, G. Ertl, and T. Matsushima, Surf. Sci. 117, 257 (1982).

${ }^{52}$ See for instance the TPD spectra for $\mathrm{H}$ on Pt from Refs. 49, 50, and 3.

${ }^{53}$ C. M. Mate and G. A. Somorjai, Phys. Rev. B 34, 7417 (1986).

${ }^{54}$ J. C. Slater, Quantum Theory of Molecules and Solids, The Self Consistent Field for Molecules and Solids, Vol. 4 (McGraw-Hill, New York, 1974).

${ }^{55}$ J. P. Perdew, in Electronic Structure of Solids, edited by P. Ziesche and H. Eshrig (Akademie Verlag, Berlin, 1991).

${ }^{56}$ J. P. Perdew, J. A. Chevary, S. H. Vosko, K. A. Jackson, M. R. Pederson, D. J. Singh, and C. Fiolahis, Phys. Rev. B 46, 6671 (1992).

${ }^{57}$ V. Milman, B. Winkler, J. A. White, C. J. Pickard, M. C. Payne, E. V. Akhmatskaya, and R. H. Nobes, Int. J. Quantum Chem. 77, 895 (2000). 